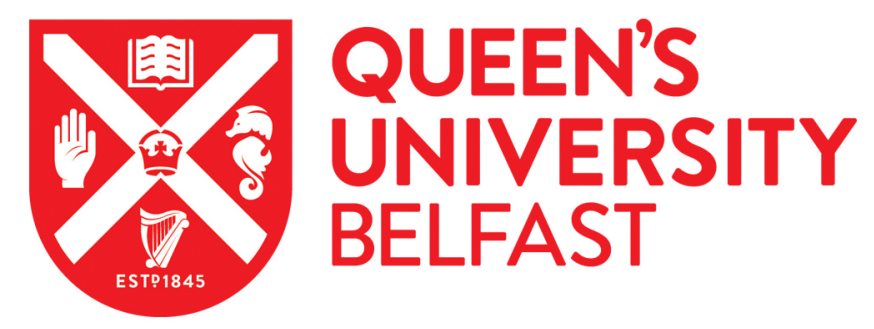

\title{
The B-type binaries characterization programme I. Orbital solutions for the 30 Doradus population
}

Villaseñor, J. I., Taylor, W. D., Evans, C. J., Ramírez-Agudelo, O. H., Sana, H., Almeida, L. A., de Mink, S. E., Dufton, P. L., \& Langer, N. (2021). The B-type binaries characterization programme I. Orbital solutions for the 30 Doradus population. Monthly Notices of the Royal Astronomical Society, 507(4), 5348-5375.

https://doi.org/10.1093/mnras/stab2197

Published in:

Monthly Notices of the Royal Astronomical Society

Document Version:

Publisher's PDF, also known as Version of record

Queen's University Belfast - Research Portal:

Link to publication record in Queen's University Belfast Research Portal

Publisher rights

Copyright 2021 The Author(s) Published by Oxford University Press on behalf of Royal Astronomical Society.

This work is made available online in accordance with the publisher's policies. Please refer to any applicable terms of use of the publisher.

\section{General rights}

Copyright for the publications made accessible via the Queen's University Belfast Research Portal is retained by the author(s) and / or other copyright owners and it is a condition of accessing these publications that users recognise and abide by the legal requirements associated with these rights.

Take down policy

The Research Portal is Queen's institutional repository that provides access to Queen's research output. Every effort has been made to ensure that content in the Research Portal does not infringe any person's rights, or applicable UK laws. If you discover content in the Research Portal that you believe breaches copyright or violates any law, please contact openaccess@qub.ac.uk. 


\title{
The B-type binaries characterization programme I. Orbital solutions for the 30 Doradus population
}

\author{
J. I. Villaseñor ${ }^{\circledR},{ }^{1 \star}$ W. D. Taylor, ${ }^{2}$ C. J. Evans, ${ }^{1,2}$ O. H. Ramírez-Agudelo ${ }^{\circledR},{ }^{3}$ H. Sana ${ }^{\circledR}, 4$ \\ L. A. Almeida ${ }^{\circledR}, 5,6$ S. E. de Mink ${ }^{\circledR},{ }^{7,8,9}$ P. L. Dufton ${ }^{10}$ and N. Langer ${ }^{11,12}$ \\ ${ }^{1}$ Institute for Astronomy, University of Edinburgh, Royal Observatory, Blackford Hill, Edinburgh EH9 3HJ, UK \\ ${ }^{2}$ UK Astronomy Technology Centre, Royal Observatory, Blackford Hill, Edinburgh EH9 3HJ, UK \\ ${ }^{3}$ German Aerospace Center (DLR), Institute for the Protection of Terrestrial Infrastructures, Rathausallee 12, D-53757 Sankt Augustin, Germany \\ ${ }^{4}$ Institute of Astrophysics, KU Leuven, Celestijnenlaan 200D, B-3001 Leuven, Belgium \\ ${ }^{5}$ Escola de Ciências e Tecnologia, Universidade Federal do Rio Grande do Norte, Natal, RN 59072-970, Brazil \\ ${ }^{6}$ Departamento de Física, Universidade do Estado do Rio Grande do Norte, Mossoró, RN 59610-210, Brazil \\ ${ }^{7}$ Max-Planck-Institut für Astrophysik, Karl-Schwarzschild-Straße 1, D-85740 Garching bei München, Germany \\ ${ }^{8}$ Anton Pannekoek Institute for Astronomy, University of Amsterdam, Science Park 904, NL-1098 XH Amsterdam, the Netherlands \\ ${ }^{9}$ Harvard-Smithsonian Center for Astrophysics, Harvard University, 60 Garden St, Cambridge, MA 02138, USA \\ ${ }^{10}$ Astrophysics Research Centre, School of Mathematics \& Physics, Queen's University, Belfast BT7 1NN, UK \\ ${ }^{11}$ Argelander-Institut für Astronomie, Universität Bonn, Auf dem Hügel 71, D-53121 Bonn, Germany \\ ${ }^{12}$ Max-Planck-Institut für Radioastronomie, Auf dem Hügel 69, D-53121 Bonn, Germany
}

Accepted 2021 July 20. Received 2021 July 20; in original form 2021 January 18

\begin{abstract}
We present results from the B-type binaries characterization (BBC) programme, a multi-epoch spectroscopic study of 88 early B-type binary candidates in the 30 Doradus region of the Large Magellanic Cloud (LMC). From radial-velocity analysis of 29 observational epochs, we confirm the binary status of 64 of our targets, comprising 50 SB1 and 14 SB2 B-type binaries. A further 20 systems (classified as SB1*) show clear signs of periodicity but with more tentative periods. Orbital solutions are presented for these 84 systems, providing the largest homogeneous sample to date of the binary properties of early B-type stars. Our derived orbital-period distribution is generally similar to those for samples of more massive (O-type) binaries in both the LMC and the Galaxy. This similarity with the properties of the more massive O-type binaries is important as early B-type stars are expected to account for the majority of core-collapse supernovae. Differences in the period distributions of the different samples start to increase above $4 \mathrm{~d}$, and are also present between the earliest (B0-0.7) and later-type (B1-2.5) systems within the BBC sample, although further study is required to understand if this is an observational bias or a real physical effect. We have examined the semi-amplitude velocities and orbital periods of our sample to identify potential candidates that could hide compact companions. Comparing with probability distributions of finding black hole companions to OB-type stars from a recent theoretical study, we have found 16 binaries in the higher probability region that warrant further study.
\end{abstract}

Key words: binaries: spectroscopic - stars: early-type - stars: massive - open clusters and associations: individual: 30 Doradus Magellanic Clouds.

\section{INTRODUCTION}

Studies in the Galaxy and the Large Magellanic Cloud (LMC) over the past decade have confirmed that the majority of massive stars are members of binary or multiple systems (e.g. Kobulnicky \& Fryer 2007; Mason et al. 2009; Chini et al. 2012; Kiminki \& Kobulnicky 2012; Sana et al. 2012, 2013, 2014; Kobulnicky et al. 2014; Dunstall et al. 2015). Much of the work done on the properties of these massive binaries has targeted O-type stars as these have the most extreme physical parameters. With masses ranging from $\sim 16 \mathrm{M}_{\odot}$ up to well over $200 \mathrm{M}_{\odot}$ (Martins, Schaerer \& Hillier 2005; Crowther et al. 2010, 2016), these stars drive the chemical evolution of their host

^E-mail: jvi@roe.ac.uk galaxies by enriching the interstellar medium (ISM) with chemically processed material from their strong winds and by exploding as corecollapse supernovae (CCSN). They also have strong ultraviolet fluxes which are capable of ionizing large volumes of gas in the ISM.

In contrast, B-type stars have received less attention. Although less exotic than O-type stars, they are substantially more numerous. The spectral range between $\mathrm{B} 0$ and $\mathrm{B} 3$ (with initial masses of $\sim 6-15$ $\mathrm{M}_{\odot}$ ) deserves special attention as they account for the majority of CCSN progenitors $(\sim 70$ per cent if assuming single-star evolution with a Kroupa 2001 initial mass function), ending their lives as neutron stars (NSs). B-type binaries, after one of the stars exploded as an SN, might become high-mass X-ray binaries (HMXB; Reig 2011). Since the discovery of the first pulsar in a binary (Hulse \& Taylor 1975), HMXBs have been proposed as a channel to produce binary NSs (BNSs; see e.g. Flannery \& van den Heuvel 1975; Tauris 
et al. 2017). Furthermore, BNSs are thought to be progenitors of interesting phenomena when merging, such as short gamma-ray bursts (Fong \& Berger 2013; Berger 2014; Abbott et al. 2017b), magnetars (Duncan \& Thompson 1992; Dai et al. 2006; Rowlinson et al. 2013), and the recently confirmed gravitational waves (Abbott et al. 2017a) and their optical counterparts, kilonovae (Coulter et al. 2017; Smartt et al. 2017).

Notably, the most numerous class of HMXBs are Be/X-ray binaries (Reig 2011; Tauris et al. 2017), where Be stars are the optical companions of the NS. We can also anticipate similar systems, where the B-type (or Be) star was originally the secondary but now appears as the primary, accompanied by an X-ray quiet compact object. Langer et al. (2020) recently computed a large grid of evolutionary models to investigate the role of mass transfer and mergers of massive binaries $\left(M_{1}=10-40 \quad \mathrm{M}_{\odot}\right)$ in forming OB-type stars with a $\mathrm{BH}$ companion. They found that the masses of OB-type stars at the moment of formation of the $\mathrm{BH}$ are mostly concentrated between 8 and $25 \mathrm{M}_{\odot}$ with a peak near $14 \mathrm{M}_{\odot}$. They also predicted 120 $\mathrm{OB}+\mathrm{BH}$ binaries in the $\mathrm{LMC}$, from which about half would be $\mathrm{B}+\mathrm{BH}$ binaries (mostly Be stars). However, only one $\mathrm{O}+\mathrm{BH}$ system is known in the LMC to date (LMC X-1, Orosz et al. 2009), arguing for a large number of $\mathrm{OB}+\mathrm{BH} / \mathrm{NS}$ systems that are $\mathrm{X}$-ray dim and potentially undetected in existing studies of OB-type stars.

Past efforts to study the multiplicity characteristics of large samples of Galactic B-type stars (Abt \& Levy 1978; Wolff 1978; Levato et al. 1987; Abt, Gomez \& Levy 1990; Raboud 1996) were mostly focused on late B-type stars and therefore are not representative of the population of CCSN progenitors. The intrinsic distributions of orbital periods for early B-type eclipsing binaries (EBs) in the Galaxy, LMC, and Small Magellanic Cloud were investigated photometrically by Moe \& Di Stefano $(2013,2015)$, with a wider review given by Moe \& Di Stefano (2017). However, there remains a lack of spectroscopic studies with sufficient cadence to determine orbital solutions for large samples of early-type binaries (e.g. Duchêne \& Kraus 2013).

Here, we present results from the B-type binaries characterization (BBC) programme (P.I. Taylor, 096.D-0825), which was conceived to better characterize the properties of binaries in the important early Btype domain. This builds on work from the VLT-FLAMES Tarantula Survey (VFTS; Evans et al. 2011), which observed more than 800 OB-type massive stars (with $V<17 \mathrm{mag}$ ) in the 30 Doradus region of the $\mathrm{LMC}$, one of the brightest and most active star-forming regions in the Local Group.

With a focus on multiplicity, six epochs of VFTS observations were used to estimate the intrinsic binary fraction of the population of O- (Sana et al. 2013) and B-type stars (Dunstall et al. 2015). Both studies found a high multiplicity fraction after bias correction; $51 \pm 4$ per cent for the O-type stars and $58 \pm 11$ per cent for the B-type stars (in good agreement within the uncertainties) for periods of up to about $8 \mathrm{yr}\left(10^{3.5} \mathrm{~d}\right)$. However, more comprehensive monitoring was required to characterize the orbital properties of the candidate binaries. With that intention, the Tarantula Massive Binary Monitoring project (TMBM) obtained 32 epochs of spectroscopy for an unbiased subset of the O-type binary sample of the VFTS. From analysis of these data, Almeida et al. (2017) presented orbital solutions for 82 systems together with the distributions of their orbital parameters. The BBC observations were designed to enable similar follow-up of 88 of the candidate B-type binaries in the VFTS from Dunstall et al. (2015).

This article is structured as follows: Section 2 describes the observational sample, Section 3 outlines our methods to estimate the stellar radial velocities (RVs), orbital periods, and determine full orbital solutions, Section 4 presents our results and compares them with published distributions for O-type stars, Section 5 discussed further aspects of our results, and Section 6 gives a brief summary of our findings.

\section{OBSERVATIONS}

\subsection{Background}

The VFTS observed 438 B-type stars, with spectral classifications and estimates of stellar RVs presented by Evans et al. (2015). In parallel, a detailed RV variability analysis was undertaken by Dunstall et al. (2015), who employed a cross-correlation technique to look for RV shifts between the (typically six) FLAMES-Giraffe spectra of each target obtained with the LR02 setting (spanning 3960$4564 \AA$ ). The spectra of all but 30 targets were sufficiently good to enable estimates of RVs, yielding results for 361 dwarfs and giants, and 47 supergiants.

The statistical criteria used to assess the RV variability of the Otype stars in the VFTS (Sana et al. 2013) were adapted by Dunstall et al. (2015) to investigate the RVs of the B-type spectra. For a target to be considered as a candidate binary system, the difference between at least one pair of RVs had to be larger than four times the combined uncertainty of the two measurements and simultaneously exceed their adopted threshold of $\Delta \mathrm{RV}_{\min }=16 \mathrm{~km} \mathrm{~s}^{-1}$. Objects with statistically significant variations in the range $5<\Delta \mathrm{RV}<16 \mathrm{~km} \mathrm{~s}^{-1}$ that fulfilled the first condition were considered as 'RV variables' (see Dunstall et al. for further discussion on selection criteria).

They found 96 candidate single-lined binaries (SB1s) and five double-lined (SB2) systems. These comprised 90 unevolved objects (i.e. the dwarfs and giants) and 11 supergiants, giving observed spectroscopic binary fractions of 25 and 23 per cent, respectively. In addition, 23 unevolved stars and 17 supergiants were classified as RV variables.

\subsection{Monitoring campaign}

The approach for the BBC campaign was to observe as many as possible of the 101 candidate binaries from Dunstall et al. in one FLAMES fibre configuration. The input list also included seven further targets that were flagged by Dunstall et al. as candidate binaries but where absolute RVs could not be estimated (see their footnote 4). The FLAMES Fibre Positioning Observation Support Software (FPOSS) was used to maximize the number of targets assigned to fibres from the 108 candidate systems, resulting in observations of the 88 systems listed in Table B1. ${ }^{1}$

To obtain coverage of a selection of hydrogen, helium, and metallic lines for RV estimates of each target, we adopted the same strategy as the TMBM campaign in using the Medusa mode of FLAMES with the LR02 set-up. This provides a spectral resolving power $R=$ 7000 , which is sufficient to obtain individual RVs precise to better than $5 \mathrm{~km} \mathrm{~s}^{-1}$.

To securely identify cosmic rays, each 1-hr observing block (OB) was comprised of three back-to-back science exposures of $894 \mathrm{~s}$. These were observed over the period 2015 October to 2016 December, where execution of the 29 OBs from the service queue was (loosely) constrained to ensure a varying cadence (from dailyintervals up to the long baseline of observations more than one year

\footnotetext{
${ }^{1}$ Comprised of 78 of the 96 candidate SB1s from Dunstall et al., four of the previously classified SB2 systems, and six of the seven additional candidates without absolute RVs.
} 
Table 1. Heliocentric Julian Dates at the start of each observing block for the BBC campaign.

\begin{tabular}{cccc}
\hline OB & HJD & OB & HJD \\
\hline 01 & 2457299.745 & 16 & 2457417.563 \\
02 & 2457332.733 & 17 & 2457418.611 \\
03 & 2457335.782 & 18 & 2457420.622 \\
04 & 2457339.698 & 19 & 2457421.695 \\
05 & 2457339.780 & 20 & 2457423.598 \\
06 & 2457366.724 & 21 & 2457427.607 \\
07 & 2457379.551 & 22 & 2457432.517 \\
08 & 2457394.652 & 23 & 2457622.866 \\
09 & 2457398.723 & 24 & 2457651.760 \\
10 & 2457400.577 & 25 & 2457681.710 \\
11 & 2457402.602 & 26 & 2457692.743 \\
12 & 2457410.692 & 27 & 2457698.748 \\
13 & 2457411.579 & 28 & 2457724.707 \\
14 & 2457415.547 & 29 & 2457726.710 \\
15 & 2457416.559 & & \\
\hline
\end{tabular}

after the start of the programme). The Heliocentric Julian Dates (HJDs) of the start of each triplet of exposures are listed in Table 1.

With the Medusa fibres assigned to the candidate binaries, we used the remaining fibres to obtain further observations of other notable VFTS targets. These included candidate O-type runaways (Walborn et al. 2014), candidate B-type runaways (Evans et al. 2015), the extreme rotator VFTS 102 (Dufton et al. 2011), the X-ray bright VFTS 399 (Clark et al. 2015), the peculiar B[e]-like supergiant VFTS 698 (Dunstall et al. 2012), and several stars of interest in the context of the nitrogen abundances from Grin et al. (2017). Although not the focus of this article, details of these additional targets are given in Table B7.

\subsection{Data reduction}

The data were reduced using the ESO CPL FLAMES/GIRAFFE pipeline v.2.1.5 for bias and dark subtraction, correction for the flatfield response, wavelength calibration, and (summed) extraction of the spectra. Each spectrum was then corrected to the heliocentric frame. An error spectrum was also produced by the pipeline for each fibre, which records the statistical error arising from different stages in the reduction for each wavelength bin.

Mean spectra were obtained for each epoch by combining the three exposures weighted by the associated error spectra. A $5 \sigma$-clip was also included when taking the average to reject outlying cosmic rays. The combined data for each epoch were then normalized by division of a low-order polynomial fit to the continuum.

All but six of our OBs were obtained in grey or dark time (fractional lunar illumination of $<70$ per cent). With the exception of two OBs $(12,13)$, the background continuum level was generally low in the dozen sky fibres allocated across the FLAMES field. We experimented with subtracting the median sky spectrum for each epoch from each science spectrum. Although it led to a marginal improvement in $\mathrm{S} / \mathrm{N}$, it then led to complications with over/undersubtraction of the nebular emission lines because some of the sky fibres had (weak) nebular emission. Given our approach to model the nebular emission as part of our RV analysis (see Section 3.1.2) and for a consistent approach to all of the spectra we decided not to subtract the sky spectra from our data. From tests with and without sky subtraction for objects without significant nebular emission, this did not have a significant impact on the final RV estimates. The exceptions were OBs 12 and 13, where they were only useful for estimates for a couple of the brightest stars. Subtracting the sky spectra for these two OBs alone does improve their $\mathrm{S} / \mathrm{N}$ but then caused problems with the nebular components, so we did not pursue this further. We add that two OBs $(10,18)$ had generally low $\mathrm{S} / \mathrm{N}$ for many of the targets due to other observational factors; these were also only used for some of the brighter targets.

\subsection{The B-type binary candidates}

The spatial distribution of the B-type sample observed by the BBC campaign in the 30 Doradus region is shown in Fig. 1; the luminosity class (LC) of each target is also shown. The central cluster, NGC 2070, contains a quarter of the observed sample, including 27 per cent of the dwarfs (LC V-IV) and two of the five supergiants (LC I). In contrast, only seven dwarfs and two giants (LC III-II) are within NGC 2060, the older association to the southwest. The two older, smaller stellar clusters, Hodge 301 and SL 639, contain only two and one BBC targets, respectively. Most of the stars in our sample are field stars (62 per cent), comprising 60 per cent of our dwarf targets and 67 per cent of the giants in the field, plus an additional supergiant and two stars without LCs.

The distribution of $V$-band magnitudes and spectral types for the sample are shown in Fig. 2, with their LC colour coded as in Fig. 1. Our B-type systems are dominated by faint $(V \sim 16-17 \mathrm{mag})$ mainsequence (MS) stars (72 per cent of the sample), with typical S/N ratios of below 40 for most of our spectra (see Section 3.1.3). Systems over the spectral range B0.5 to B2.5 are well distributed, but there is a drop at the earliest subtypes, with only nine members in the B0-0.2 bin (of which two do not have an LC). For the latest types (B3-5), dwarfs were too faint for the VFTS magnitude limit $(V=17$, see Evans et al. 2011), so only three giants/bright giants were observed.

From visual inspection of the spectra and the subsequent RV analysis, we noted 14 targets as SB2 (or candidate SB2) systems, of which half were previously identified. The SB2s thus comprise 16 per cent of the sample, lower than the 33 per cent fraction of binaries that were SB2s in the TMBM sample. This is probably (partly) a consequence of the fainter magnitudes of the B-type stars and hence the lower $\mathrm{S} / \mathrm{N}$.

\section{METHODOLOGY}

\subsection{Stellar radial velocities}

Following the approach taken by Sana et al. (2013) and Almeida et al. (2017), we determined RVs from shifts of the spectral lines due to the Doppler effect. We have employed a line-by-line Gaussian fitting method to each spectrum that uses LMFIT (Newville et al. 2014), a non-linear least-squares minimization package available in PYTHON.

The dominant absorption lines in the LR02 region of early Btype spectra are the Balmer series and a set of He I lines. These are complemented by a range of weak metallic features (e.g. Si III, Si IV, and $\mathrm{Mg}$ II) and, at the earliest types, weak He II absorption. Of the metallic lines, given the $\mathrm{S} / \mathrm{N}$ of our spectra and the distribution of spectral types, only Si III $\lambda 4553$ offers a useful RV diagnostic for a reasonable number of our targets. Si IV is only present at the earliest types (and blended with an O II line in the case of Si IV $\lambda$ 4089), while $\mathrm{Mg}$ II is only present in the latest types.

For the majority of our sample we therefore used seven lines to estimate the RVs: $\mathrm{H} \delta, \mathrm{H} \gamma$, He I $\lambda \lambda 4026,4144,4388,4471$, and Si III $\lambda 4553$. For the small number of stars classified earlier than B0.5, we also attempted fits to the He II $\lambda \lambda 4200,4542$ lines, but these did 


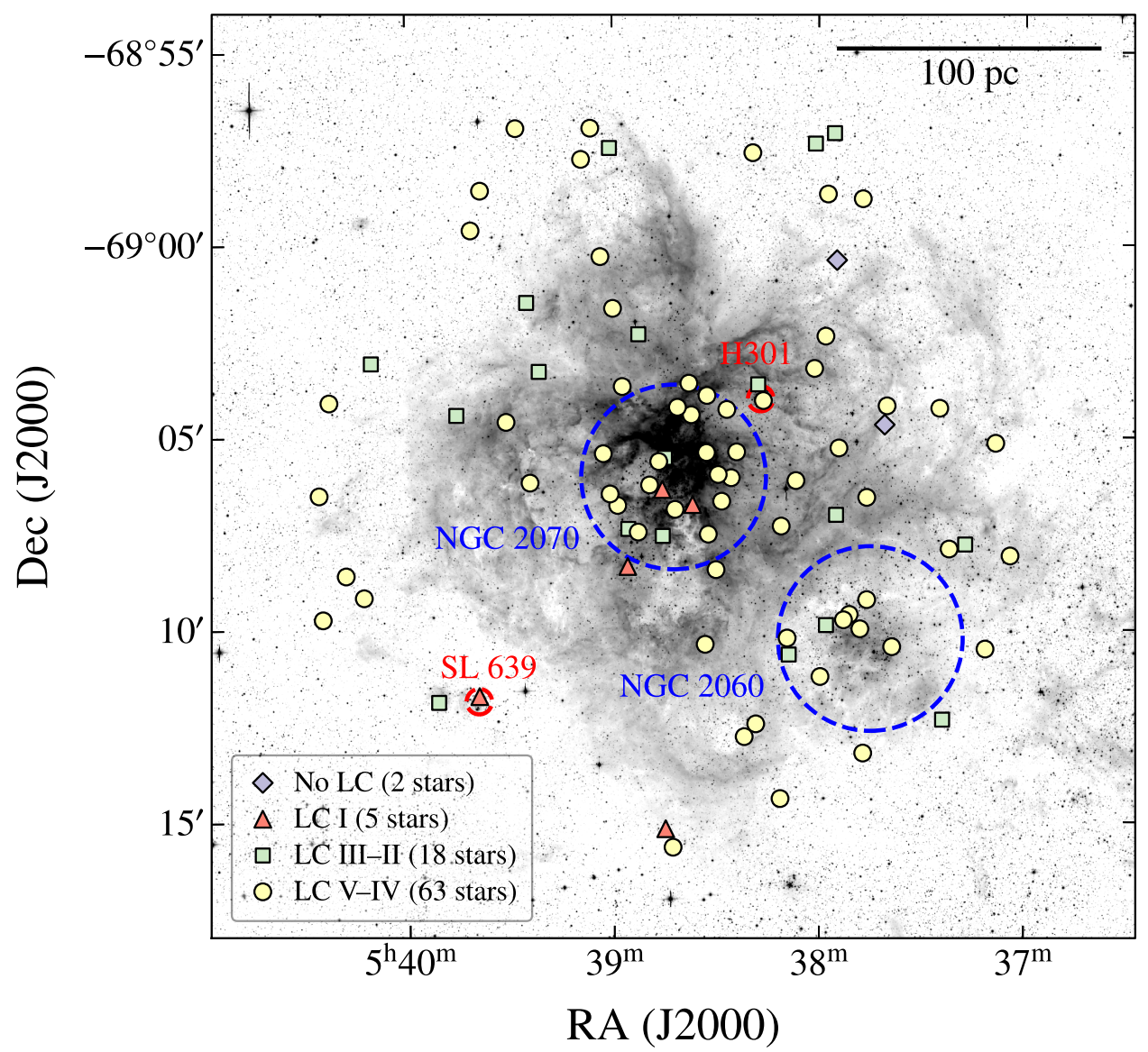

Figure 1. Spatial distribution of the 88 B-type binary candidates with new spectroscopy. The sample has been plotted by LC (see legend). The blue-dashed circles (with radii of 2'.4) highlight the approximate extent of the NGC 2070 and NGC 2060 clusters, whereas SL 639 and Hodge 301 (with radii of 0'33) are represented in red.
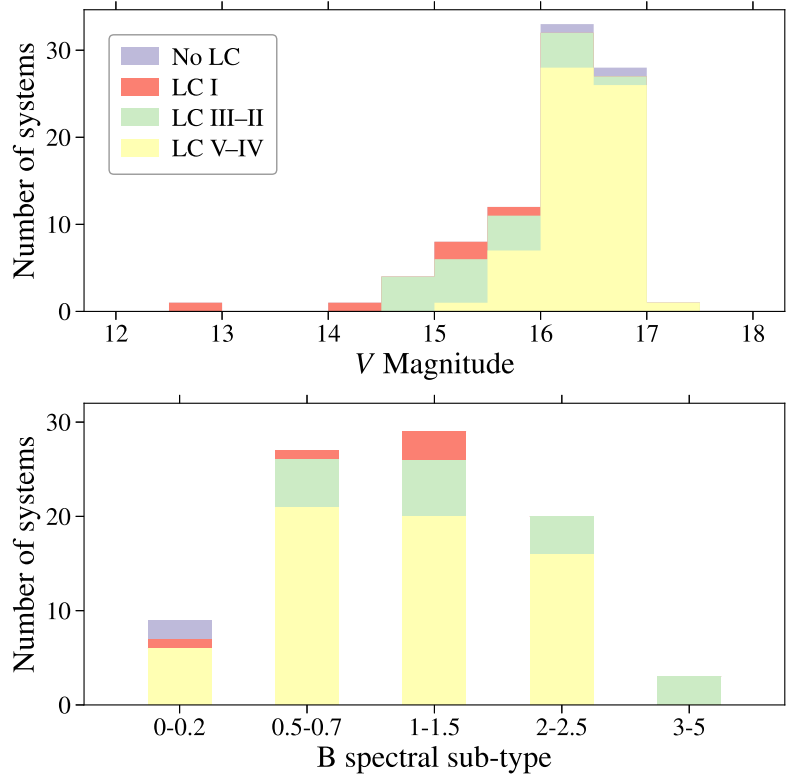

Figure 2. Histograms of $V$-band magnitudes (upper panel) and spectral types (lower panel) of the BBC sample (spectral classifications from Evans et al. 2015).

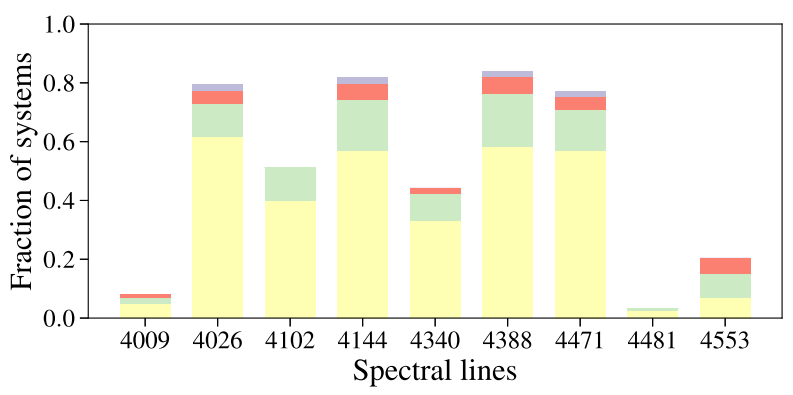

Figure 3. Fraction of systems for which each spectral line was used to estimate RVs. The colours are as in Figs 1 and 2.

not yield useful information in most cases given the weakness of the features combined with the $\mathrm{S} / \mathrm{N}$ of the spectra from each epoch. In general, the weaker $\mathrm{He} I(\lambda \lambda 4009,4121)$ lines were too weak to provide useful fits. However, for six systems where we otherwise struggled to find a period, the $\mathrm{He}$ I $\lambda 4009$ and $\mathrm{Mg}$ II $\lambda 4481$ lines were strong enough to provide useful information and led to improved results.

To ensure a sufficient number of lines for each target it was also necessary to separately take into account the nebular contamination (see Section 3.1.2). The fraction of the sample for which each diagnostic line was used in estimating the final RVs of each target is shown in Fig. 3. 
The line fits for the RV estimates were based on a set of given initial parameters. Each line was fitted from an initial estimate of the centre $(\mu)$, amplitude $(A)$, and full width at half-maximum (FWHM), employing Gaussian profiles for the helium and silicon lines, and a Lorentzian for the Balmer lines. In the case of slow rotators (mainly evolved stars), the profiles of the He I lines will be dominated by the instrument profile which is Gaussian, whereas for rapid rotators the spectral line profile is dominated by rotational broadening which is not Gaussian. However, the $\mathrm{S} / \mathrm{N}$ of our sample tends to be lower for MS stars (i.e. generally the faster rotators), so the line profile can be fitted sufficiently well by Gaussians. We tested both Gaussian and Lorentzian fits to the spectra of stars with different LCs finding that the Gaussian fits did a better job of fitting the wings of the lines in all cases.

All the lines were fitted independently for each epoch of each star, obtaining values and uncertainties for $A, \mu$, and FWHM. A median-absolute-deviation (MAD; see e.g. Hampel 1974) test was then applied to these uncertainties to identify the diagnostic lines with better fits (smaller errors) for each system. Given that the errors on $\mu$ and $A$ were typically smaller, the FWHM errors were used in most cases for the MAD test, as these proved to be more sensitive to erroneous fits (e.g. misidentification of the line due to low $\mathrm{S} / \mathrm{N}$, solar features, or intrinsic weakness of the spectral line). The MAD test is a robust measure of dispersion in a data set and is commonly used to find and reject outliers. It is defined as the median of the absolute differences of the data from their median:

$\operatorname{MAD}(X)=k \operatorname{med}(|X-\operatorname{med}(X)|)$,

where med stands for median, $X$ is a variable denoting the data values, and $k$ is a 'consistency' constant dependant on the distribution. Here, we have assumed a normal underlying distribution for our errors for which $k$ takes a value of 1.4826 (Rousseeuw \& Croux 1993). A rejection criterion $(\mathrm{RC})$ must be set such that:

$\frac{X-\operatorname{med}(X)}{\operatorname{MAD}(X)}>\mathrm{RC}$

is considered an outlier. In our case, the medians of the uncertainties for the fits in the 29 epochs are computed for each spectral line and the MAD test is applied to them; spectral lines with a value given by equation (1) larger than the adopted RC are considered outliers and therefore rejected. An RC value between 2 and 3 is usually used, with a higher value being a more relaxed outlier cutoff. The remaining set of lines was then used to compute the RV of each observation.

With the lines selected, the individual spectra were also checked to reject spectra with low $\mathrm{S} / \mathrm{N}$ (see Section 3.1.3) to avoid undue influence of misidentifications of line centres on the final RV estimate. Erroneous fits can be identified by their characteristically large errors, so an MAD test similar to that used to select the diagnostic lines was applied to detect epochs with poor fits. For each epoch, a mean uncertainty was computed for each fitted parameter considering the lines selected in the previous step. The MAD test again searches for outliers in the mean uncertainties, i.e. epochs with large errors, and their RVs were not included in the computation of the orbital period. Fig. 4 shows the number of spectral lines (top) and number of epochs (bottom) used in the RV analysis (with the SB1 and SB2 systems highlighted). For $\sim 80$ per cent of the systems at least four lines (up to a maximum of seven) were used, while the number of epochs fluctuates around a mean of 24, with a strong peak at 25. Fewer epochs were used for the SB2 systems, with a mean of only 15 (see Section 3.1.1).
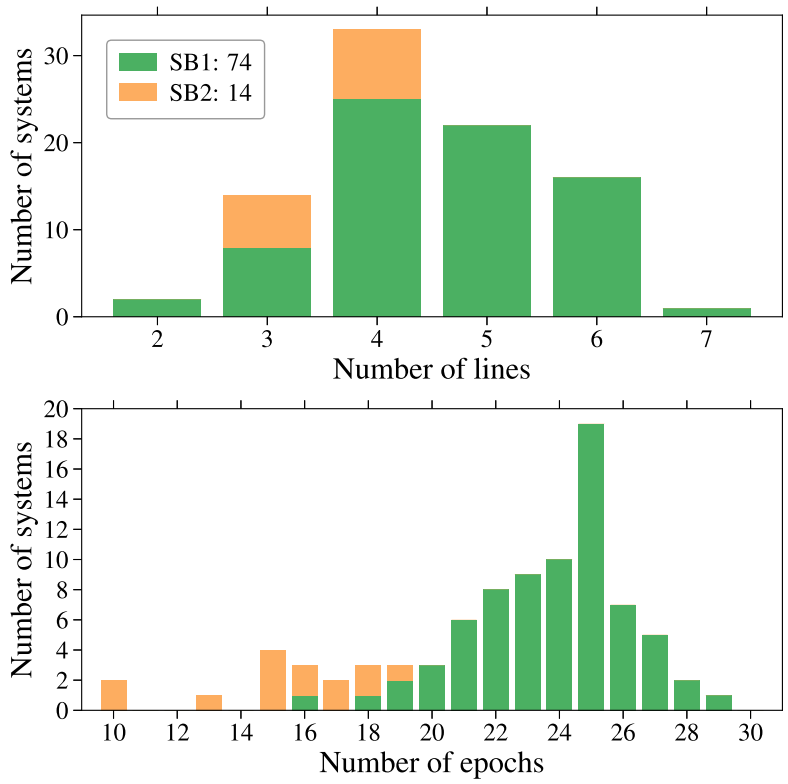

Figure 4. Top panel: Number of spectral lines used to determine RVs for each system. Bottom panel: Final number of epochs used to determine the orbital periods after removing epochs with low $\mathrm{S} / \mathrm{N}$ or with large $\mathrm{RV}$ errors. In both panels, the SB2 systems are shown in orange.

Table 2. Absorption lines used in the determination of RVs with their respective rest wavelengths from the NIST Atomic Spectra Database (Kramida et al. 2020). ${ }^{2}$

\begin{tabular}{lll}
\hline Ion & $\lambda(\AA)$ & $\lambda_{\text {rest }}(\AA)$ \\
\hline He I & 4009 & 4009.256 \\
$\mathrm{He} \mathrm{I}$ & 4026 & 4026.191 \\
$\mathrm{H} \delta$ & 4102 & 4101.734 \\
$\mathrm{He} \mathrm{I}$ & 4144 & 4143.761 \\
$\mathrm{H} \gamma$ & 4340 & 4340.472 \\
$\mathrm{He} \mathrm{I}$ & 4388 & 4387.930 \\
$\mathrm{He} \mathrm{I}$ & 4471 & 4471.480 \\
$\mathrm{Mg}$ II & 4481 & 4481.130 \\
$\mathrm{Si} \mathrm{III}$ & 4553 & 4552.620 \\
\hline
\end{tabular}

RVs $\left(v_{\text {rad }}\right)$ were calculated for the selected epochs and lines from:

$v_{\text {rad }}=\frac{\mu-\lambda_{\text {rest }}}{\lambda_{\text {rest }}} c$

where the adopted values of $\lambda_{\text {rest }}$ are given in Table 2 and $c$ is the speed of light. For each epoch of each system, a weighted mean was calculated from the RVs of the selected spectral lines using

$\bar{v}_{\mathrm{r}}=\frac{\sum_{i} \omega_{i} v_{i}}{\sum_{i} \omega_{i}}$ with $\omega_{i}=\frac{1}{\left(\sigma_{i}\right)^{2}}$,

where $v_{i}$ are the individual RVs calculated from each of the spectral lines available, while $\omega_{i}$ are the weights chosen as the inverse of the uncertainty squared that LMFIT computes from the covariance matrix.

\footnotetext{
${ }^{2}$ https://physics.nist.gov/PhysRefData/ASD/lines_form.html
} 


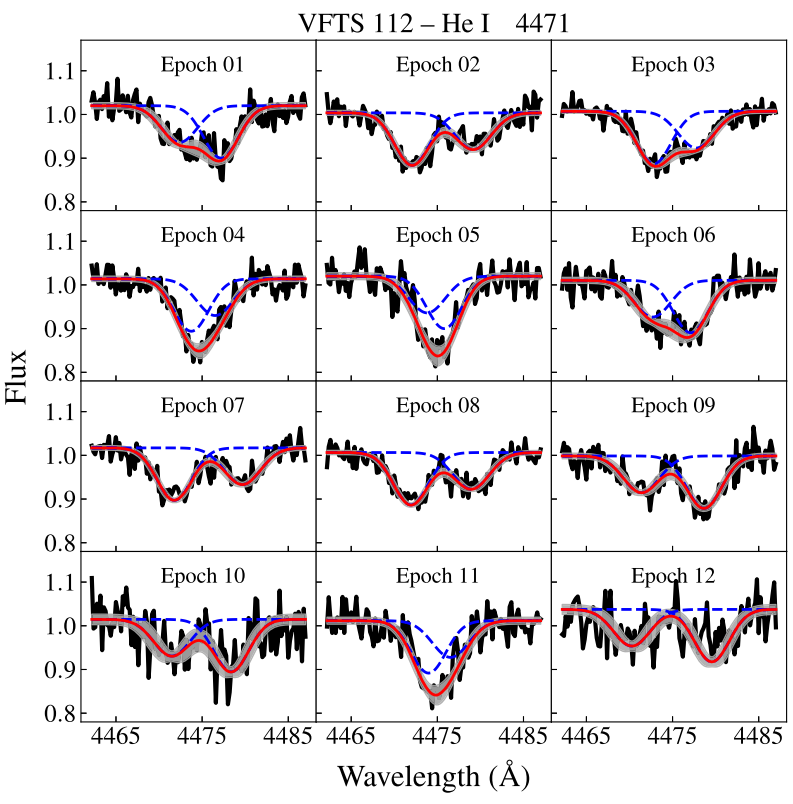

Figure 5. Example fits for the SB2 system VFTS 112. Blue-dashed lines indicate individual spectral lines for primary and secondary, red-solid lines are the resulting fits, with the grey-shaded areas representing the $3 \sigma$ uncertainties.

The uncertainty of the RV weighted mean was calculated from

$\sigma_{\bar{v}_{\mathrm{r}}}=\frac{1}{\sum_{i} \omega_{i}} \sqrt{\sum_{i}\left(\omega_{i} \sigma_{i}\right)^{2}}$,

which follows from the definition of variance and equation (4). The choice of a weighted mean was to include as many epochs as possible, to exploit any useful information we can from the lower S/N observations.

\subsubsection{Double-lined systems}

Double Gaussian fits were implemented in the code to measure the RVs of both components of the identified SB2 systems. A key difference with the (single-line) fits for the SB1 systems is when the $\mathrm{RV}$ difference of the two components is close to zero. This impacts on the quality of the fit as it becomes difficult to identify the contribution of each star to the blended spectral line, as shown by epochs 4,5 , and 11 in Fig. 5. To avoid erroneous RVs from such observations, the code uses a minimum separation for the components (of 2 to $4 \AA$ A, depending on the line width) below which epochs are rejected. The rejected epochs will therefore be those with RVs close to the systemic velocity, i.e. where the velocities of the two components intersect in the final orbital solution (see Fig. C3, available as supplementary material).

In some spectra the two components can be separated sufficiently well, but the code occasionally misidentifies the two components, e.g. if the primary is redshifted, the code can associate the blueshifted line to the primary and vice versa. Effects such as low S/N spectra and systems with comparable intensities of the two components are the main cause of such misidentifications. Thus, the code checks for discrepancies in the shifts of the primary component in an epoch. For example, for a set of four spectral lines, if the primary (stronger) component is redshifted in three and blueshifted in the remainder, the code inverts the primary and secondary component in the discrepant line, so that the primary is now redshifted in all four cases. However, if the fits to the primary component were redshifted and blueshifted



Figure 6. Fraction of systems for key diagnostic lines where the nebular contamination was sufficiently bad that the RV analysis included a model emission component (see Section 3.1.2 for details).

the same number of occasions (two each in the example), it is not possible to distinguish both components and the code rejects that epoch. One last check acts on noisy observations, where the code applies an MAD test to the errors of the calculated centre of the lines, rejecting spectra with large errors, as with the SB1 systems.

These three filters reduced the number of observations used to estimate the orbital period for the SB2 systems to an average of 15 epochs (in contrast to an average of $\sim 24$ for the SB1 systems, see Fig. 4). The majority of the SB2 systems are short-period binaries, thus we expect the orbits to be mostly circular or to have small eccentricities. This means that a robust estimate of the period is possible with fewer epochs in the case of SB2 systems, and that little information is lost regarding the eccentricity as long as the rejected epochs are close to the systemic velocity and the separation between $\mathrm{RV}$ measurements is not extreme.

\subsubsection{Nebular contamination}

Nebular contamination of the spectra varies significantly, from being completely absent in some cases to very strong in a large fraction of the sample. This is most notable in the Balmer lines, where the nebular emission can be several times stronger than the stellar absorption. Fig. 6 shows the frequency of nebular contamination for our diagnostic lines. The Balmer lines of nearly all of our targets displayed some degree of contamination and $\sim 60$ per cent of the He I $\lambda 4471$ profiles are also significantly affected. It is less of a problem for the other helium lines, with only 20,10 , and 6 per cent significantly affected for He I $\lambda 4026, \lambda 4388$, and $\lambda 4144$, respectively.

To have enough lines for robust RV estimates, it was necessary to include the Balmer lines and to account for nebular emission. We fitted the spectra with a superposition of a Gaussian/Lorentzian profile (for the contaminated He I/Balmer absorption lines) and of a Lorentzian profile (nebular and/or Be-type stellar disc emission). In other words, we fit both the spectral absorption line and the nebular/disc emission, as shown by the example in Fig. 7. Due to the variation in the relative strength of the nebular emission between epochs (arising from variations in the astronomical seeing impacting differently on point and extended sources in terms of the flux at the fibre aperture), it was not possible to develop an automated method of detection, so the lines affected by nebulosity were identified by eye.

\subsubsection{Signal to noise}

A second difficulty with our adopted methods was the low $\mathrm{S} / \mathrm{N}$ of many of the spectra as mentioned in Section 2.4, including variations in the $\mathrm{S} / \mathrm{N}$ of repeated observations of each target given changes in the observing conditions, airmass, lunar illumination, etc. The $\mathrm{S} / \mathrm{N}$ 


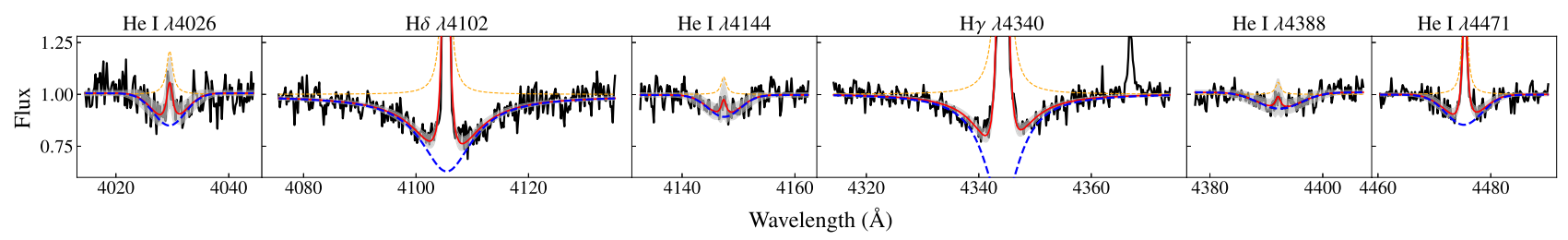

Figure 7. Example fits for VFTS 337 that take into account contamination by nebular emission. Although classified as a Be star (B2: V-IIIe+ from Evans et al. 2015), from inspection of the [O III] and [N II] lines in the LR03 and HR15N data from the VFTS, the nebular emission dominates the Be contribution. The two components, stellar absorption (blue) and nebular emission (orange), are shown together with the resulting fit (red) to illustrate our procedure, and the varying degree of contamination in the different lines.

per pixel of our spectra are generally in the range of 15 to 60 , with the exception of the supergiant VFTS 591 for which the spectra have $\mathrm{S} / \mathrm{N} \sim 120$. The mean $\mathrm{S} / \mathrm{N}$ of the sample is 33 (with a median of 30 ), with 83 per cent of the spectra having $\mathrm{S} / \mathrm{N}<40$ and 53 per cent with $\mathrm{S} / \mathrm{N}<30$, down to a minimum of $\mathrm{S} / \mathrm{N} \sim 15$. This is even more pronounced in the dwarfs, with all of the spectra having $\mathrm{S} / \mathrm{N}<50$ and 65 per cent with $\mathrm{S} / \mathrm{N}<30$. Although low $\mathrm{S} / \mathrm{N}$ increases the challenge of successful fits to the spectra (and to identify SB2 systems), it has less impact on the period search (see discussion in Section 5.1.2).

\subsection{Orbital periods}

The Lomb-Scargle (LS) periodogram (Lomb 1976; Scargle 1982) is a widely used tool in the astronomical community to search for signals in unevenly sampled time-series. We implemented the LS routines from ASTROPY (Astropy Collaboration 2013; Price-Whelan et al. 2018) to search for orbital periods within the weightedmean RVs for each epoch of each system. The BBC programme was designed to find periods between one day and a year, but a frequency grid covering a range between 0.4 and $1000 \mathrm{~d}$ was used for the LS analysis because three systems showed broad peaks in the periodogram close to $500 \mathrm{~d}$. We tested our code on three B-type systems that were also observed as part of the TMBM programme (VFTS $225,779,827$ ) and found differences of less than 0.4 per cent between our estimated periods and those from Almeida et al. (2017).

To determine the significance of the periods found, we computed the false-alarm probability (FAP) to estimate the probability of having a peak in the periodogram of a certain height at a given frequency if the data show no periodic signal. While the FAP does not tell us if we are correctly identifying the true period of the system, it is a measurement of the significance of the signal (VanderPlas 2018). We considered a peak as corresponding to the true period if its FAP exceeded a false-alarm level (FAL) of 0.1 per cent. Systems that satisfy this condition and that also have an orbital solution were classified as SB1.

Beyond these cases, there were further systems that show clear signals in their periodograms. These include cases with an FAP between 0.1 and 1 per cent (or just below 0.1 per cent), and some examples with double peaks of similar strength. Even if they present convincing orbital solutions with a low $\chi^{2}$, to differentiate them from those with more robust periods, we classified them as SB1*. Periodograms for each of the systems classified as SB1, SB1*, and SB2 are available as supplementary material in Appendix C.

A last group of systems from our targets are those with FAPs larger than 1 per cent and low LS powers $(\$ 2)$. We could not find a reliable orbital period for these targets and they were classified as RV variables (RV var). Nevertheless, the possible orbital periods obtained for the RV var systems are given in Table 3, together with
Table 3. Velocity results for the four targets classified as RV variables, i.e. with significant $\mathrm{RV}$ shifts but uncertain periods.

\begin{tabular}{lccc}
\hline VFTS & $\Delta \mathrm{RV}_{\max }\left(\mathrm{km} \mathrm{s}^{-1}\right)$ & $\sigma_{\mathrm{RV}}\left(\mathrm{km} \mathrm{s}^{-1}\right)$ & Possible $P_{\text {orb }}(\mathrm{d})$ \\
\hline 144 & $58.77 \pm 8.05$ & 9.78 & 171.37 \\
391 & $60.25 \pm 10.24$ & 10.21 & 1.12 \\
591 & $25.44 \pm 2.02$ & 0.89 & 478.68 \\
890 & $35.23 \pm 3.74$ & 5.23 & 18.92 \\
\hline
\end{tabular}



Figure 8. Classification of the SB1 systems. The ratio of the LS power at the 0.1 per cent FAL to the peak value ( $P_{\text {peak }}$, used to identify the potential period) for each system as a function of $P_{\text {peak }}$. Systems with $P_{\text {peak }}$ above the 0.1 per cent FAL therefore have a ratio $<1$ (i.e. a strong signal), which defines the green area with robust periods ( 73 per cent of the sample). The yellow area includes 15 systems with less certain periods that are classified as SB1* (with some exceptions outside this area from considering other factors). Systems that did not exceed the 1 per cent FAL were classified as RV variables and are indicated in red.

maximum $R V$ variation $\left(\Delta \mathrm{RV}_{\max }\right)$ and standard deviation of the mean $\mathrm{RV}\left(\sigma_{\mathrm{RV}}\right)$. These periods should only be considered as tentative at best but, considering the significant RV variations in some of them, it is possible that these are the true period and other factors are interfering with the signal in the periodogram (see Section 5.1).

Our classification criteria are illustrated in Fig. 8. The $x$-axis corresponds to the LS power of the peak in the periodogram and the $y$-axis is the ratio of the power at 0.1 per cent FAL and the power of the peak (i.e. the value in the $x$-axis). The green-shaded area indicates a region with LS power $\geq 4$ and above 0.1 per cent FAL (or $\mathrm{P}_{\mathrm{FAL}} / \mathrm{P}_{\text {peak }}<1$ ). A total of 64 binaries, including all the SB2 systems, are in this region (i.e. 73 per cent of the $\mathrm{BBC}$ sample). The 


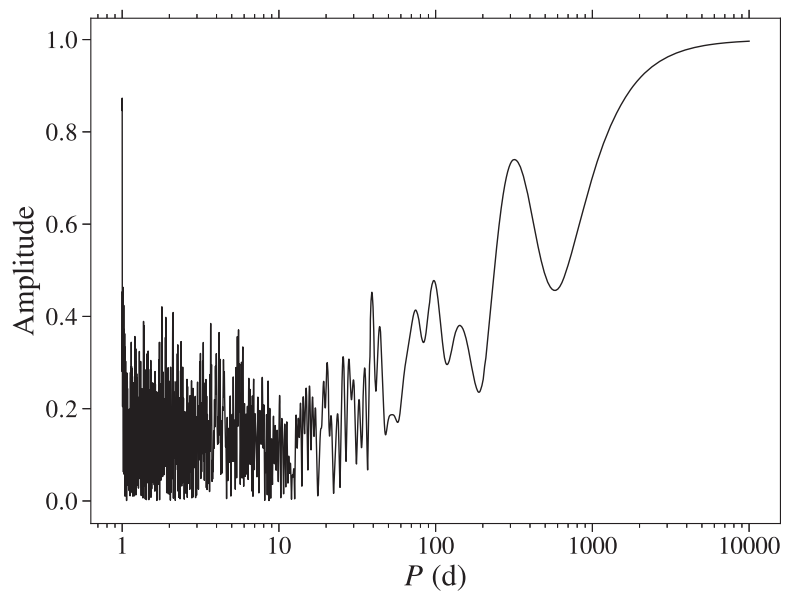

Figure 9. Power spectrum of the BBC observing window. Our observational strategy enabled us to determine orbital periods ranging from one day up to a year.

labelled objects are the exceptions to our criteria, but we emphasize these are not strict limits and their binary classification took into account orbital solutions, morphology, and other aspects. For notes on the individual systems, see Appendix A.

\subsubsection{Non-sinusoidal signals}

The LS periodogram searches for a period by fitting a sinusoidal model to each frequency of the grid. Of course, the best model will not always be a sinusoid, e.g. due to eccentricity of the orbit. Nevertheless, given our time-sampling, we expect most of our systems to be relatively short-period binaries with near-circular orbits. For example, 60 per cent of the O-type systems observed by the TMBM programme had periods of less than $20 \mathrm{~d}$, and 40 per cent had eccentricities of less than 0.1 (Almeida et al. 2017).

For eccentric systems, where the signal is not sinusoidal, higher harmonics are expected to be present in the periodogram. For a system with a true period $\left(P_{\text {true }}\right)$ at a frequency $f_{0}$, higher harmonics could be present at $m f_{0}$, with $m$ a positive integer (VanderPlas 2018). However, this will not prevent the sinusoidal model finding the correct period if it can closely fit the data.

\subsubsection{Other aliasing effects}

The power spectrum of our observing window is shown in Fig. 9 where two clear peaks are visible. The larger one above $300 \mathrm{~d}$ corresponds to the $1 \mathrm{yr}$ alias and the peak at $1 \mathrm{~d}$ can be explained as a consequence of the day and night cycle. Such features can create aliases at $f_{0} \pm n \delta f$, where $\delta f$ in the case of nocturnal observations (typical of ground-based observatories) would be 1 cycle $\mathrm{d}^{-1}$ and $n \in \mathbb{Z}^{+}$(see VanderPlas 2018, and references therein). We have identified such aliases in most of our sample and they have been taken into account when determining the periods. The power spectrum demonstrates that we have very good detectability for periods of up to $50 \mathrm{~d}$. Beyond this our detectability declines, which significantly limits the accuracy of periods above $100 \mathrm{~d}$, while becoming increasingly difficult or infeasible to detect periods up to the baseline of the BBC programme ( $427 \mathrm{~d}$ ). Periods longer than this cannot be trusted since not even a full cycle is covered.

\subsubsection{Checks on the orbital periods}

Most of our adopted lines for RV estimates are affected by Stark broadening and three of our helium lines are diffuse. To test the robustness of the orbital periods determined by our LS analysis, we implemented a brute-force method that takes all the available lines in each spectrum and combines them to form subsets of a minimum of three lines (two in a few difficult cases and the SB2s). If we take all nine lines in Table 2, this gives 466 possible sets of lines. We then tested each of the combinations with the LS routine, to estimate the fraction of the different combinations that returned the same (or a different) period from our initial analysis. This method proved useful to identify weak (but robust) signals in some of the systems where we had otherwise struggled to estimate a period, as well as confirming the majority of the periods of the SB1 systems.

\subsection{Orbital solutions}

Full orbital solutions were obtained for the complete sample with the IDL code RVFIT (Iglesias-Marzoa, López-Morales \& Jesús Arévalo Morales 2015). RVFIT uses an adaptive simulated annealing algorithm (Ingber 2000) to fit Keplerian orbits to RV data using seven parameters: orbital period $P_{\mathrm{orb}}$, time of periastron passage $T_{p}$, eccentricity $e$, argument of the periastron $\omega$, systemic velocity $\gamma$, semi-amplitude of the primary $K_{1}$ and, in the case of SB2 systems, the semi-amplitude of the secondary star $K_{2}$. The code minimizes the $\chi^{2}$ function:

$\chi^{2}=\sum_{i=1}^{N}\left[\left(\frac{\tilde{v}_{1}\left(t_{i}\right)-v_{1}\left(t_{i}\right)}{\sigma_{1}\left(t_{i}\right)}\right)^{2}+\left(\frac{\tilde{v}_{2}\left(t_{i}\right)-v_{2}\left(t_{i}\right)}{\sigma_{2}\left(t_{i}\right)}\right)^{2}\right]$,

where $N$ is the number of RV measurements, $v_{1}$ and $v_{2}$ are the RVs of the primary and secondary stars (when available) at time $t_{i}$ and $\sigma_{1}$ and $\sigma_{2}$ are their associated uncertainties. Finally, $\tilde{v}_{1,2}$ is the RV the code computes by solving the following set of equations:

$$
\begin{aligned}
& \tilde{v}_{1}\left(t_{i}\right)=\gamma+K_{1}\left[\cos \left(\theta\left(t_{i}\right)+\omega\right)+e \cos \omega\right] \\
& \tilde{v}_{2}\left(t_{i}\right)=\gamma+K_{2}\left[\cos \left(\theta\left(t_{i}\right)+\omega^{\prime}\right)+e \cos \omega^{\prime}\right] \\
& \omega^{\prime}=\omega+\pi \\
& \theta\left(t_{i}\right)=2 \arctan \left[\sqrt{\frac{1+e}{1-e}} \tan \left(\frac{E\left(t_{i}\right)}{2}\right)\right] \\
& E\left(t_{i}\right)-e \sin E\left(t_{i}\right)=M\left(t_{i}\right) \\
& M\left(t_{i}\right)=\frac{2 \pi}{P}\left(t_{i}-T_{p}\right),
\end{aligned}
$$

where $\theta$ is the true anomaly, $E$ is the eccentric anomaly which must be obtained by solving Kepler's equation (equation 11), $M$ is the mean anomaly and $T_{p}$ is the time of periastron passage. Once RVFIT selects the best model from the minimization of the $\chi^{2}$, it returns the orbital parameters with their respective uncertainty, which are computed from the covariance matrix (see Iglesias-Marzoa et al. for details).

We folded the RVs to the estimated periods from the LS tests $\left(P_{\text {orb }}\right)$ and fitted sinusoids to the RV curves. From the sinusoidal fits we obtained initial values for $\gamma, K_{1}$, and $K_{2}$ (for SB2s), in which we used the median value of the HJDs of our observations as the initial parameter for $T_{p}$. We then used these as input to the RVFIT analyses, adopting a search range of \pm 20 per cent on the initial values for $P_{\text {orb}}$, $\gamma, K_{1}$, and $K_{2}$, while $T_{p}$ was constrained by the initial and end dates of the observing campaign. For $e$ and $\omega$, we used initial values of 0.1 
and 0 , respectively, letting them vary in (almost) the full parameter space (0-0.999 for $e$ and $0-360^{\circ}$ for $\left.\omega\right)$.

The estimated periods from the RVFIT analyses are in excellent agreement with the LS results, with a median difference of only 0.04 per cent (when comparing to the initial LS periods) for the whole sample. We also found small differences for the other parameters, with a median of 0.2 per cent for $\gamma$ and 5.6 per cent for $K_{1}$, where the latter was expected to be larger given the inclusion of eccentricity. Results for all the orbital parameters are given in Table B3 for the SB1s, in Table B4 for the SB1* systems, and in Table B5 for SB2s. The minimum masses, mass ratios, and minimum orbital separations of the SB2 systems are listed in Table B6. RV curves obtained from solutions given by RVFIT are available as supplementary material for all systems in Appendix D.

\section{RESULTS}

In summary, from the analysis of the 88 candidate B-type binaries observed by the BBC programme, we have found reliable periods for 64 binaries and found clear signs of periodicity for 20 further systems (i.e. estimated periods for 95 per cent of the sample). The remaining four systems display RV variations but without significant periodic signals. We now describe our results and compare them with published results for other samples of massive binaries.

\subsection{Distribution of orbital parameters}

\subsubsection{Orbital periods}

The observed distribution of orbital periods is shown in Fig. 10 (top panel). The number of short-period SB1 systems increases from four systems with $P_{\text {orb }}<2$ d, up to 11 with $8<P_{\text {orb }}<16$ d. The SB2 sample substantially increases the number of binaries with $P_{\text {orb }}<$ $8 \mathrm{~d}$, making the distribution almost constant in $\log$ space up to $P_{\text {orb }} \sim 16 \mathrm{~d}$. This range presents a large accumulation of systems, in fact the majority of the BBC sample are short-period binaries; from the cumulative distribution function (black circles in Fig. 10), 40 per cent of the BBC sample have $P_{\text {orb }}<5 \mathrm{~d}$, 75 per cent have $P_{\text {orb }}<30 \mathrm{~d}$, and $\sim 85$ per cent have $P_{\text {orb }}<100 \mathrm{~d}$. The SB1* group increases the number of systems with short periods $(P<2 \mathrm{~d})$ but includes more systems in the range 10-100 d (to be expected given that it becomes harder to secure robust detections of longer periods with the cadence of our observations).

To determine if the contribution of the SB1* systems is statistically significant we have compared the distribution of SB1+SB2 systems with respect to the full sample $\left(\mathrm{SB} 1+\mathrm{SB} 2+\mathrm{SB} 1^{*}\right)$ with a k-sample Anderson-Darling (AD) test and a 2-sample Kuiper (K) test. The $\mathrm{AD}$ test statistic returned a negative value which was lower than the critical value for the 25 per cent significance level (at which the value is capped) meaning that the results are not significant at a significance level of 25 per cent, so we cannot reject the null hypothesis that the two samples are drawn from the same distribution. This is supported by the $\mathrm{K}$ test which returned a false-positive probability (fpp) value of 0.997 , i.e. there is a K-test probability of 99.7 per cent of obtaining two samples this different from the same distribution. These results are summarized in Table 4. Given the similarity of the two distributions, we use the SB1+SB2 distribution (i.e. black circles in Fig. 10) in the following discussion.
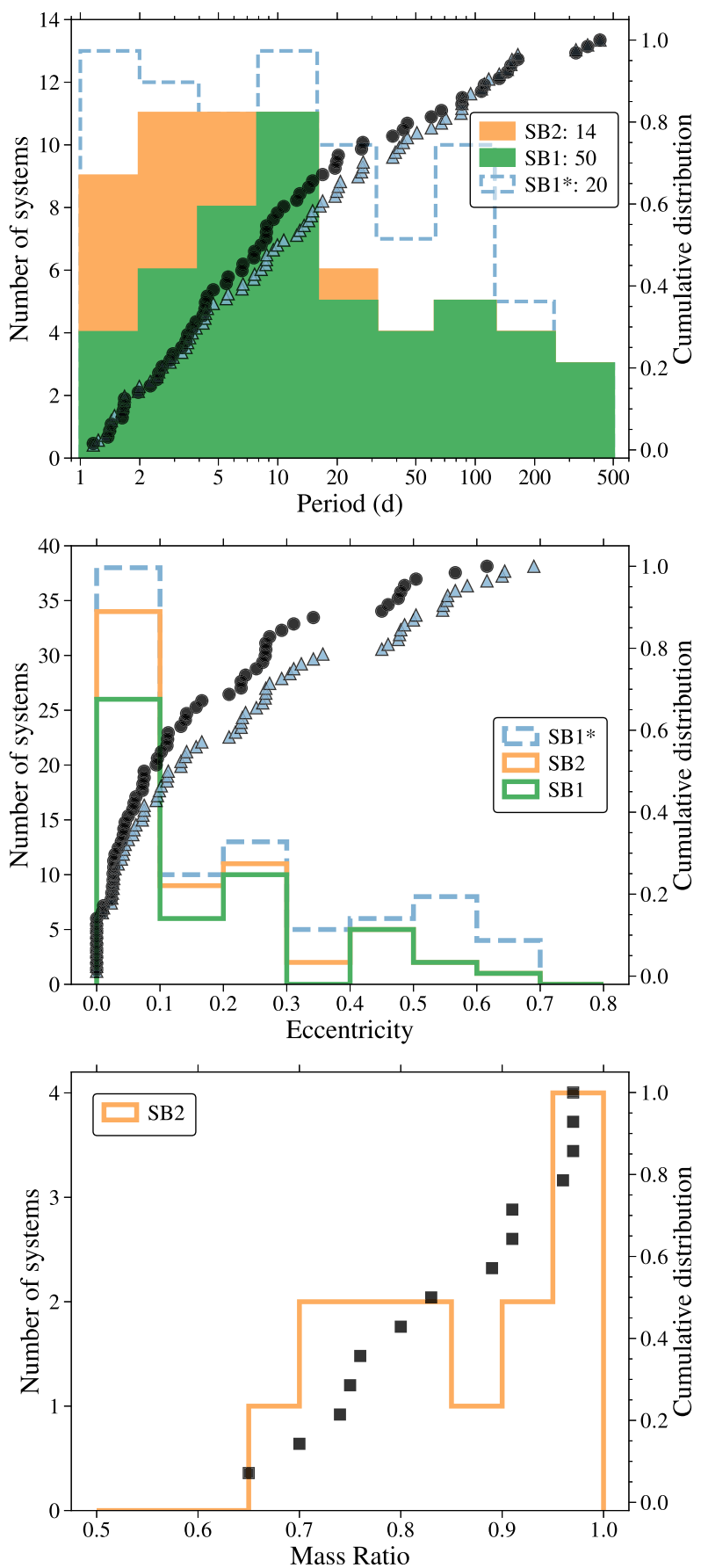

Figure 10. Distribution of orbital parameters $\left(P_{\mathrm{orb}}, e, q\right)$ for the SB1 and SB2 systems; the dashed-blue histograms include systems with possible but unconfirmed periods (SB1*). The cumulative distributions of the combined SB1 and SB2 systems are indicated by black circles in the upper two panels, while the blue triangles show the distributions with the addition of the SB1* systems. Black squares in the lower panel show the cumulative distribution of mass ratios for the SB2 systems.

\subsubsection{Eccentricities}

Results for eccentricities $(e)$ are also shown in the middle panel of Fig. 10. There is a concentration of systems with $e<0.1$ ( 55 per cent of the sample), confirming that many of the binaries have circular or close-to-circular orbits. Fig. 11 shows that low eccentricities $(e$ $<0.2)$ are preferred by the shorter period systems $\left(P_{\text {orb }}<20 \mathrm{~d}\right)$. 
Table 4. Results from Anderson-Darling and Kuiper tests on the distributions of orbital parameters from the BBC, and other Galactic and LMC samples of O- and B-type binaries.

\begin{tabular}{llrrrr}
\hline Sample 1 & Sample 2 & \multicolumn{2}{c}{ AD test } & \multicolumn{2}{c}{ K test } \\
& & Stat & SL (\%) & D & fpp (\%) \\
\hline
\end{tabular}

BBC subsamples

Periods:

\begin{tabular}{|c|c|c|c|c|c|}
\hline BBC & $\mathrm{BBC}+\mathrm{SB} 1^{*}$ & -0.947 & 25 & 0.110 & 99.7 \\
\hline ricitics. & & & & & \\
\hline $\mathrm{BBC}$ & $\mathrm{BBC}+\mathrm{SB} 1^{*}$ & 0.195 & 5 & 0.120 & 98 \\
\hline \multicolumn{6}{|c|}{ Luminosity class and spectral type: } \\
\hline $\mathrm{BBC}$ & Dwarfs only & -1.149 & & 0.086 & \\
\hline arly-B dwarfs & Late-B dwarfs & 1.375 & 28 & 0.427 & \\
\hline
\end{tabular}

Comparison with other OB-type samples

30 Dor O-type period distribution:

$\begin{array}{llllll}\text { BBC } & \text { TMBM } & -0.481 & 25 & 0.173 & 73.8 \\ \text { Early-B dwarfs } & \text { TMBM dwarfs } & -1.072 & 25 & 0.208 & 92.0\end{array}$

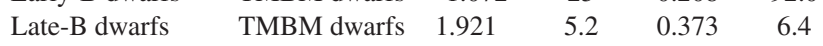

Galactic OB-type period distribution:

\begin{tabular}{|c|c|c|c|c|c|}
\hline $\mathrm{BBC}$ & $\mathrm{S} 12$ & -0.490 & 25 & 0.226 & 69.9 \\
\hline $\mathrm{BBC}$ & K14 & -0.353 & 25 & 0.221 & 55.3 \\
\hline $\mathrm{S} 12$ & TMBM & 0.872 & 14.3 & 0.311 & 16.7 \\
\hline Early-B dwarfs & S12 dwarfs & 0.449 & 21.7 & 0.376 & 24 \\
\hline Late-B dwarfs & S12 dwarfs & -0.497 & 25 & 0.267 & 73 \\
\hline \multicolumn{6}{|c|}{ Galactic B-type period distributions: } \\
\hline BBC dwarfs & K14 dwarfs & -0.024 & 25 & 0.333 & 34 \\
\hline Early-BBC & Late-K14 & -0.464 & 25 & 0.419 & 31 \\
\hline Late-BBC & Late-K14 & 3.028 & 1.90 & 0.507 & 6.7 \\
\hline \multicolumn{6}{|c|}{ Eccentricity distributions: } \\
\hline BBC & $\mathrm{S} 12$ & -0.673 & 25 & 0.239 & 10 \\
\hline BBC & K14 & 5.650 & 0.20 & 0.519 & 0.3 \\
\hline BBC & TMBM & 3.908 & 0.87 & 0.359 & 0.3 \\
\hline
\end{tabular}

Note. The 'BBC sample' here refers to the SB1+SB2 results. The four subcolumn entries are: (1) AD test statistic (Stat); (2) significance level (SL); (3) $\mathrm{K}$ test statistic (D); (4) false-positive probability (fpp).



Figure 11. Eccentricities versus orbital periods for the BBC sample. The solid-magenta line represents the curve of contact for typical B-type binaries with a combined radius $\left(R=R_{1}+R_{2}\right)$ of $10 \mathrm{R}_{\odot}$ and total mass of $23 \pm 5$ $\mathrm{M}_{\odot}$ with uncertainties represented by the grey-shaded area.
From the cumulative distribution in Fig. 10, close to 90 per cent of the detected binaries have $e<0.4$, with only two systems with $e>0.55$ and none with $e>0.65$. The systems classified as SB1* contain 10 high-eccentricity systems $(e>0.4)$ but, as shown by Fig. 11, the uncertainties on $e$ for several of these are typically larger than the rest of the sample, including three possible short-period systems close to the curve of contact for a typical B-type-star radius (which gave us further cause to classify them as SB1*). As for the orbital periods, we conducted $\mathrm{AD}$ and $\mathrm{K}$ tests (K-test probability of 98.9 per cent), which argue that the samples are not significantly different (i.e. $\mathrm{SB} 1+\mathrm{SB} 2$ versus $\mathrm{SB} 1+\mathrm{SB} 2+\mathrm{SB} 1 *$ ).

\subsubsection{Mass ratios}

The distribution of mass ratios $(q)$ for the 14 SB2 systems is shown in the lower panel of Fig. 10. There are no systems with $q<0.65$ and half the detected systems have near equal-mass components (with $q>0.90$ ). These results are not unexpected given the observational limitations where the quality of the data, and the rapid drop in luminosity for lower mass companions, makes it easier to detect near equal-mass systems. For the O-type binaries from Almeida et al. (2017), companions were detected down to $q$ as low as 0.35 , but a companion with such a small mass ratio would be undetectable with our observations of the lower mass, B-type systems.

\subsection{Comparison of spectral properties}

We investigated the period distribution of the SB1+SB2 sample with respect to spectral type and LC (serving as proxies for the mass and evolutionary status, respectively, of the primaries). The upper panel of Fig. 12 shows the cumulative period distribution for the whole sample compared with distributions grouped by LC. Our sample is dominated by dwarfs so it is difficult to say much about the more evolved systems. The giants (classes III and II) are present across most of the range of periods, with a small peak in the 5$10 \mathrm{~d}$ bin. There are only three (out of the initial five) supergiants, distributed between 8 and $50 \mathrm{~d}$ and, as one might expect given their evolutionary status, absent at the shortest periods (in which a merger or a common-envelope configuration would have already occurred given their large radii). While the number of evolved stars is too low to draw any conclusions, we have compared the full sample with that of the dwarfs using the $\mathrm{AD}$ and $\mathrm{K}$ tests and found no statistically significant difference.

To investigate the properties of the dwarfs further, the lower panel of Fig. 12 shows the period distributions for the earliest (B0-0.7) and later (B1-2.5) types in the sample. There is a notable rise of later-type systems around $4 \mathrm{~d}$, with the fraction of later-type binaries about 30 per cent higher than the earlier-type systems for $P_{\text {orb }}<$ $8 \mathrm{~d}$. Interestingly, this contrasts with the results from Almeida et al. (2017) who found a tendency towards short periods for early O-type binaries in comparison to later O-types.

Our results could be explained by a bias in being less sensitive to detection of lower mass secondaries in the later-type systems. Assuming a flat distribution of mass ratios, late-type primaries have less massive companions in comparison to early-type primaries. At longer periods, binaries with less massive secondaries will be more difficult to detect as they present smaller semi-amplitude velocities. For example, for two systems with primary masses of 14 and $8 \mathrm{M}_{\odot}$, for $q=0.3, P_{\text {orb }}=100 \mathrm{~d}, e=0$, and $i=45^{\circ}$, their semi-amplitude velocities are 19.7 and $16.3 \mathrm{~km} \mathrm{~s}^{-1}$, respectively, just above our detection limit. This small difference is unlikely 



Figure 12. Top panel: Cumulative distribution of orbital periods of the BBC sample and those for subsets by LC. (Note that there were two targets without luminosity classifications.) Bottom panel: Cumulative distribution of the MS stars split into the earliest and later B-type systems in our sample.

to explain the relative dearth of late B-type stars with longer periods on its own, but probably combines with the fact that we will be more sensitive to smaller mass ratios for the more massive primaries.

Our statistical tests indicate that these two sub-samples are different at a significance level of 9 per cent from the AD test and a probability of 13 per cent from the $\mathrm{K}$ test, so not formally significant. Another possible explanation for the later types favouring shorter periods could be that it was harder to detect longer periods for such stars from the original VFTS data, presuming they were generally fainter (i.e. lower $\mathrm{S} / \mathrm{N}$ ), such that they were not included in the BBC sample. However, the range of spectral types is relatively small, and the magnitudes of the targets in the two bins overlap significantly (e.g. due to differences in line-ofsight extinction), suggesting this might not be a strong factor in our results. Moreover, there did not appear to be a significant difference in the estimated binary fractions as a function of magnitude from the VFTS analysis (see fig. 4 from Dunstall et al. 2015, although again extinction is probably a limiting factor). We note that the observed binary fraction of B0-0.7 stars from Dunstall et al. was 32 per cent, while it was only 21 per cent for those classified B1-2.5. Further investigation beyond the scope of this study is required to ascertain if the binary fraction is truly lower in the later-type bin, or if observational factors have influenced the results.

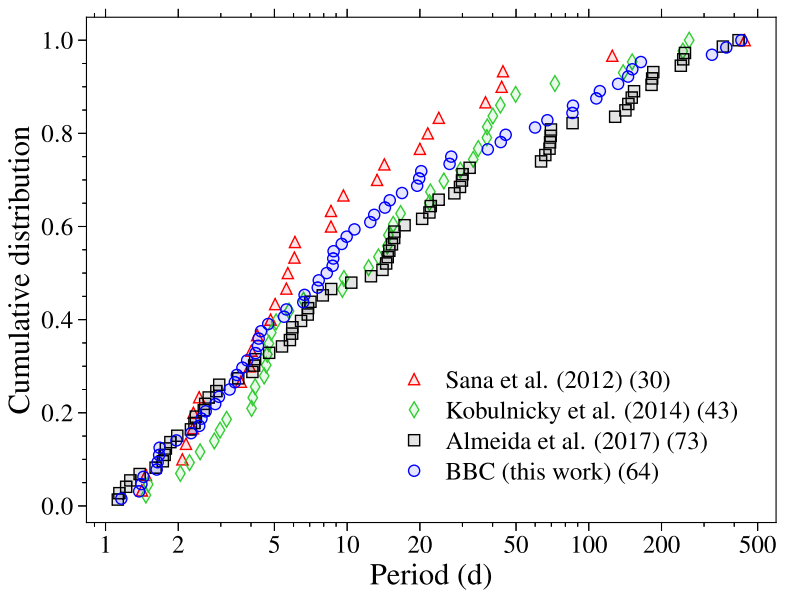

Figure 13. Cumulative distribution of orbital periods from the BBC systems (blue circles) compared with multiplicity studies of OB-type binaries in the Galaxy (Sana et al. 2012; Kobulnicky et al. 2014, red triangles and green diamonds, respectively) and the LMC (Almeida et al. 2017, black squares). The three published samples have been truncated at periods of $500 \mathrm{~d}$ to enable better comparison with the $\mathrm{BBC}$ results.

\subsection{Comparison with published samples}

In Fig. 13, we compare the orbital period distribution of our B-type binaries with those from three published studies:

(i) S12: O-type stars from Sana et al. (2012), comprised of 40 O-type (O3-9.7) binaries in six young (1-4 Myr) Galactic clusters, with the analysis based on (typically) 20 epochs of observations from previous studies.

(ii) K14: Results for 48 (23 O- and $25 \mathrm{~B}$-type) stars in the Cygnus OB2 association (3-4 Myr) from Kobulnicky et al. (2014). Their analysis was based on 14 epochs obtained at $R=2500$ to 4500 and good S/N (60 to 200), with RV estimates from He I $\lambda 5876$, and extended with observations from previous campaigns with different telescopes.

(iii) TMBM: Results for VFTS follow-up of 93 O- and 7 B-type binaries in 30 Dor in the LMC by Almeida et al. (2017), using the same FLAMES LR02 set-up as here.

Given the absence of systems with periods larger than $500 \mathrm{~d}$ in our sample, we truncated the published samples at this period for a better comparison. Each of these campaigns followed similar observational strategies but we note that the distributions do not account for the different observational biases in the final observed samples. To quantify the statistical significance of the differences seen in Fig. 13 we performed $\mathrm{AD}$ and $\mathrm{K}$ tests on the different samples. The results from these tests are included in Table 4.

\subsubsection{O-type binaries in 30 Dor}

Our first test was to compare our results for the B-type systems with those for the O-type binaries in 30 Dor from the TMBM study. Both campaigns had similar observational strategies, targeting the full population of CCSN progenitors in 30 Dor between them.

For $P_{\text {orb }}<4 \mathrm{~d}$, the distributions are near identical, although with a slightly larger fraction of the O-type binaries at the shortest periods $\left(P_{\text {orb }}<1.4 \mathrm{~d}\right)$. There is a small difference at $P_{\text {orb }} \sim 5 \mathrm{~d}$, with larger differences evident for $8<P_{\text {orb }}<30 \mathrm{~d}$. For instance, at $P_{\text {orb }}=11 \mathrm{~d}$, the $\mathrm{BBC}$ fraction is at 60 per cent while it is 48 per cent for the TMBM results. Aside from these differences, the two distributions 


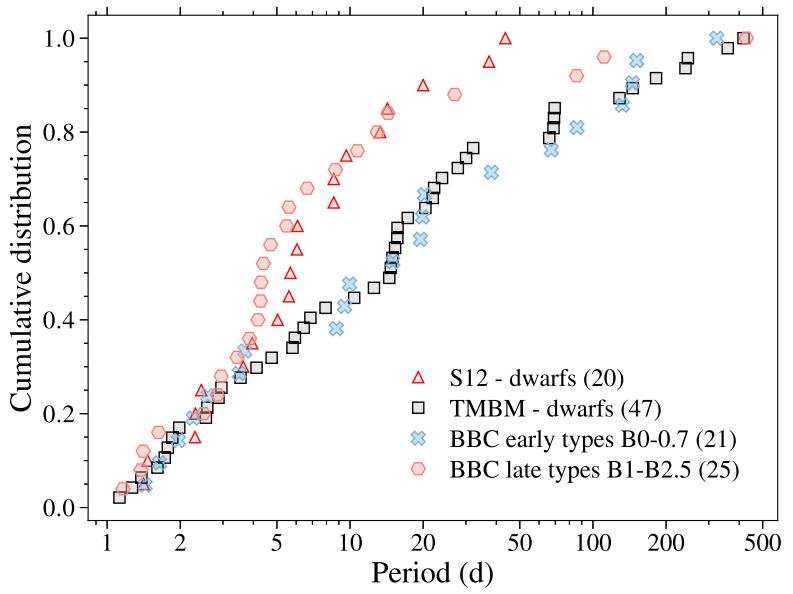

Figure 14. As in the bottom panel of Fig. 12 with the inclusion of dwarfs from the S12 and TMBM samples.

are generally similar, and both of our statistical tests found no evidence to reject the null hypothesis, with an AD-test significance level of 25 per cent and a K-test probability of 73.8 per cent.

\subsubsection{Galactic samples}

The period distributions of the published Galactic samples display more differences with the $\mathrm{BBC}$ results. At $2 \mathrm{~d}$, the O-type binaries (S12) and Cyg-OB2 (K14) samples have a lower fraction of systems, with a comparable fraction for all four samples at $P_{\text {orb }} \sim 5 \mathrm{~d}$. It is remarkable that 40 per cent of the systems in three of the samples have $P_{\text {orb }}<6 \mathrm{~d}$ (and with 55 per cent of systems from S12).

Differences between the distributions start to more clearly appear after $P_{\text {orb }} \sim 8 \mathrm{~d}$. For $P_{\text {orb }}=13 \mathrm{~d}$, the binary faction is 70 per cent for S12, 60 per cent for BBC, and more like 50 per cent for $\mathrm{K} 14$ (comparable to the TMBM fraction). These differences are reduced by $20 \mathrm{~d}$, but increase again at $\sim 40 \mathrm{~d}$, with a larger fraction of the K14 sample having $P_{\text {orb }}$ of $30-50 \mathrm{~d}$. All four samples have fractions above 80 per cent for $P_{\text {orb }}<70 \mathrm{~d}$, with both Galactic samples reaching 90 per cent by a $P_{\text {orb }} \sim 50$ d, beyond which their slopes flatten off, with relatively few systems with periods in the range of 200 to $500 \mathrm{~d}$.

As summarized in Table 4, our formal comparisons of the BBC results with those from S12 and K14 reveal no statistically significant differences. For completeness, we also compared the two O-type distributions (i.e. S12 and TMBM), finding a K-test probability of 16.7 per cent, which is lower than the value of 27 per cent from Almeida et al. (2017) that only included the O-type stars (omitting the small number of early B-type objects) and also considered the longer period systems omitted here. Similarly, the AD test returns a value of 14 per cent, which is still not statistically significant.

\subsubsection{Dwarf samples}

The Galactic and LMC period distributions of O-type dwarfs are compared with the two BBC sub-samples from Fig. 12 (i.e. B00.7 and B1-2.5) in Fig. 14. The earliest BBC dwarfs have a 92 per cent probability of being drawn from the same distribution as the TMBM sample of O-type dwarf systems. This suggests that the more massive B-type stars in the LMC follow the same period distribution as the more massive, MS (and sub-giant) O-type binaries.

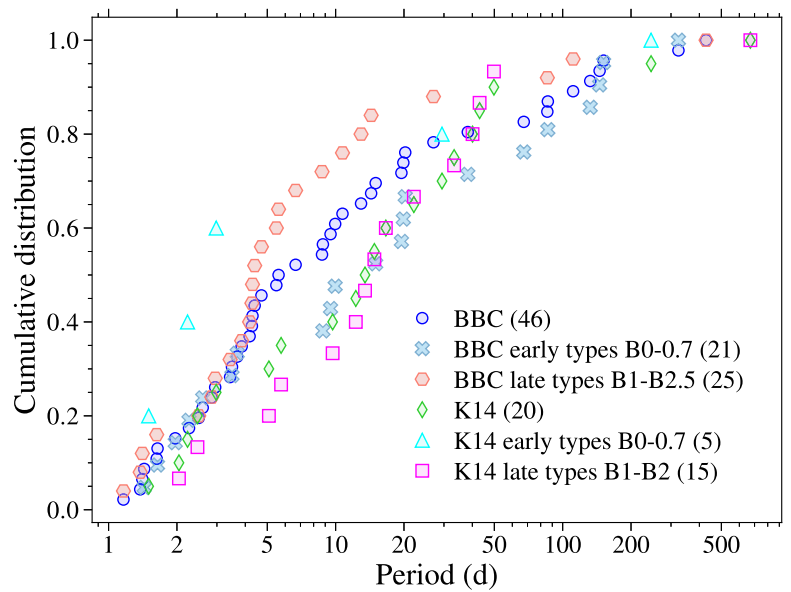

Figure 15. Comparison of cumulative distributions of periods between our sample and the B-type binaries from Kobulnicky et al. (2014). Blue circles and green diamonds represent the distributions of dwarfs for each sample. In both cases, we have separated the samples in early and later-B-type stars with almost identical spectral ranges.

In contrast, the period distribution of the later B-type binaries more closely follows the distribution of the Galactic sample of O-type binaries from S12 (K-test probability of 73.5 per cent). As discussed above, observational factors might influence the results for the latertype BBC systems, and the S12 study was not sensitive to longer period systems. More thorough investigation of the biases in the $\mathrm{BBC}$ sample to quantify the intrinsic distributions for the B-type stars will be explored in a future paper.

\subsubsection{B-type samples}

We took a closer look at the results for the B-type binaries from K14 compared to our period distribution (Fig. 15). The distributions are qualitatively quite different, with the largest difference ( $\sim 20$ per cent) at $P_{\text {orb }}=10 \mathrm{~d}$. Nonetheless, these are not statistically significant (in part given the sample sizes), with a K-test probability of 34.4 per cent of drawing these samples from the same distribution.

We also split the K14 sample into early/late types in Fig. 15 as we did earlier for the BBC results. This results in only five members in the earlier spectral bin for the K14 results, so we only consider the later-type objects further (albeit still with a limited sample size of only 15). The later-type, B-type Galactic binaries appear to more closely follow the distribution of the earlier-type systems from the $\mathrm{BBC}$ results (at least for periods of up to a month). The K-test returned a probability of 31.3 per cent between the early $\mathrm{BBC}$ and late K14 samples, which drops further to 6.7 per cent when comparing the two groups of later-type systems. However, both selection effects and small sample sizes limit meaningful conclusions.

A study of the B-type binary population of NGC 6231 (Banyard et al., in preparation) will considerably increase the number of B-type binaries characterized in young Galactic clusters, enabling a better comparison between the properties of Galactic and LMC B-type binaries in the near future.

\subsubsection{Eccentricities and mass ratios}

The distributions of eccentricities and mass ratios are shown in the upper and lower panels of Fig. 16, respectively, for the BBC results compared with the other three samples. In both figures, the 

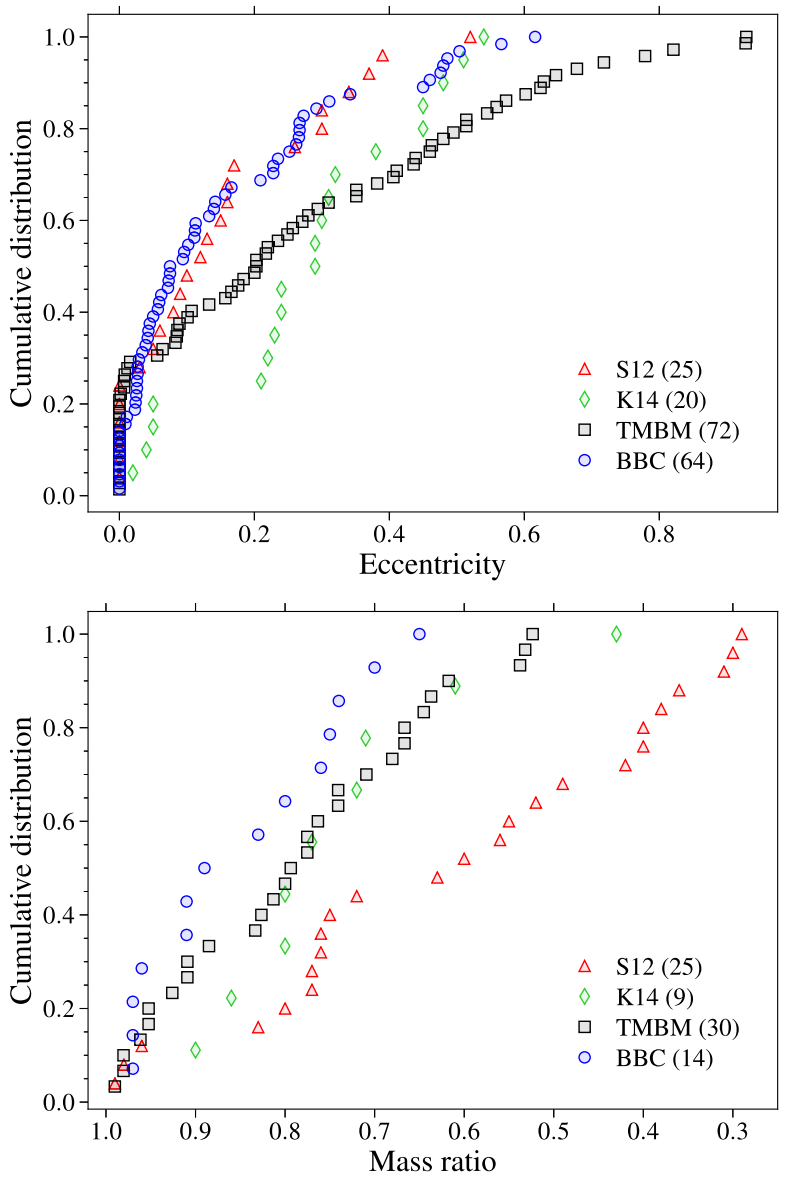

Figure 16. Cumulative distributions of eccentricities (top) and mass ratios for the BBC SB2 systems (bottom) compared with the three published samples.

K14 results are limited to only the B-type systems, and we note that S12 was not able to estimate either parameter for five of their systems.

Close to 30 per cent of all the systems have $e<0.03$, with the exception of the B-type results from K14 where 80 per cent of the systems have $0.2<e<0.6$. Once above 30 per cent, the $\mathrm{TMBM}$ distribution also differs from the $\mathrm{BBC}$ results due to the much larger eccentricities for some of the O-type systems (even some with $e>0.9$ ). For both the TMBM and K14 results, the probability of the $\mathrm{BBC}$ results being drawn from the same distribution is only 0.3 per cent. The BBC distribution is similar to that from $\mathrm{S} 12$, although our sample has a larger number with $e>0.4$ (and the S12 sample is relatively small in comparison).

Meaningful comparison of the mass ratios is not possible due to the small samples and the different observational factors influencing the B-type SB2s compared to the O-type systems (see Section 4.1.3) but we have included the distributions of all samples in the bottom panel of Fig. 16 for completeness.

\section{DISCUSSION}

\subsection{Period detectability}

We were unable to confirm the orbital period for the 20 systems that we labelled as SB1* in Section 3.2, which include five giants (three of which have spectral types of B2 or later), five (from six) of the Be-type stars, and one supergiant (see Table B4). These 20 systems constitute 23 per cent of the $\mathrm{BBC}$ sample, and there is a

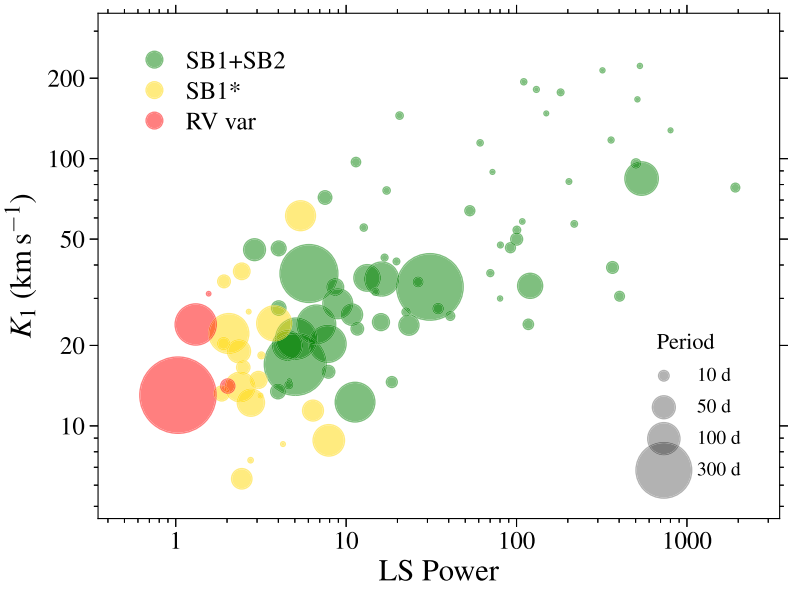

Figure 17. Comparison of semi-amplitude velocities $\left(K_{1}\right)$ and LS power. The three groups classified by the reliability of their estimated periods show that low LS powers are correlated with low $K_{1}$ values. Circle sizes are scaled by the orbital periods as shown.

further 5 per cent (four targets) for which we did not find signs of periodicity (Table 3). Possible explanations for these include low-amplitude RV shifts from low-mass companions, fast rotators, low $\mathrm{S} / \mathrm{N}$ of our targets, and that our spectral range exactly spans the temperature domain of pulsating B-type stars, e.g. $\beta$ Cep (Stankov $\&$ Handler 2005) and even slowly pulsating stars (Waelkens et al. 1998). These points are now discussed in turn.

\subsubsection{Effects of semi-amplitude velocities and rotation}

Fig. 17 offers clues for the non-negligible fraction of systems without confirmed orbital periods. There is a clear trend of semi-amplitude velocities with LS power: systems with larger $K_{1}$ present stronger signals in the periodograms and vice versa, albeit with some notable exceptions, as discussed in Appendix A. In fact, 85 per cent of the SB1* systems have $K_{1} \lesssim 30 \mathrm{~km} \mathrm{~s}^{-1}$. It is also interesting to note that the systems with larger periods $\left(P_{\text {orb }} \gtrsim 100\right)$ have LS powers of less than 20 in most cases, an indication of the difficulties in finding long-period binaries from our observational campaign.

Moreover, an analysis of the projected rotational velocities $\left(v_{e} \sin i\right.$ ) of our sample (taken from Dufton et al. 2013; Garland et al. 2017; see Table B1) shows that 40 per cent of the SB1* systems have $v_{e} \sin i>230 \mathrm{~km} \mathrm{~s}^{-1}$, from which three were classified as $\mathrm{Be}$ stars by Evans et al. (2015). Fig. 18 shows the $v_{e} \sin i$ values versus semi-amplitude velocities; all SB1* systems are either relatively fast rotators $\left(v_{e} \sin i>150 \mathrm{~km} \mathrm{~s}^{-1}\right.$ ), have low semi-amplitude velocities $\left(K_{1}<20 \mathrm{~km} \mathrm{~s}^{-1}\right)$, have high eccentricities $(e>0.4)$, or a mixture of these properties.

This evidence might suggest a few challenges: (i) for systems with low $K_{1}$, we do not have enough $\mathrm{S} / \mathrm{N}$ to accurately determine the RV shifts; (ii) small RV shifts might be more affected by stellar pulsations; (iii) an important fraction of systems with small RV shifts are high-eccentricity (and/or long-period) binaries; (iv) systems with large $v_{e} \sin i$ values might need higher $\mathrm{S} / \mathrm{N}$ to determine robust $\mathrm{RV}$ shifts and periods; $(v)$ a combination of all these points.

\subsubsection{S/N of the BBC campaign}

The lower $\mathrm{S} / \mathrm{N}$ of the BBC sample in comparison to the TMBM and Galactic samples might have limited the period estimates of some of our targets. However, this does not appear to be the case given 


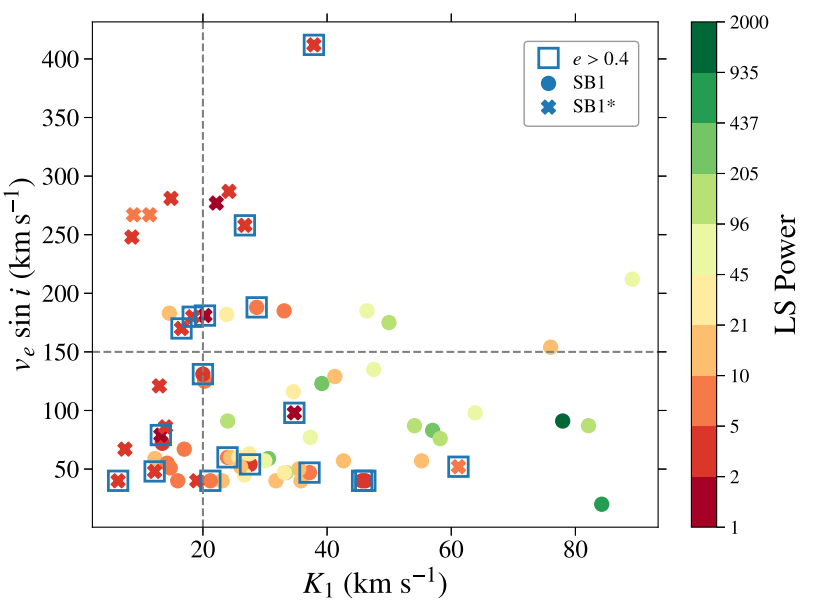

Figure 18. Rotational velocities as a function of the semi-amplitude velocities for the SB1 and SB1* samples. Systems with high eccentricities are marked by blue squares. The diagram suggests that all SB1* systems have less significant signals in the LS periodogram due to their high $v_{e} \sin i$, low $K_{1}$, and/or high eccentricities. LS power is indicated by the log-scale colour bar, with the tick labels rounded to the closest integer.

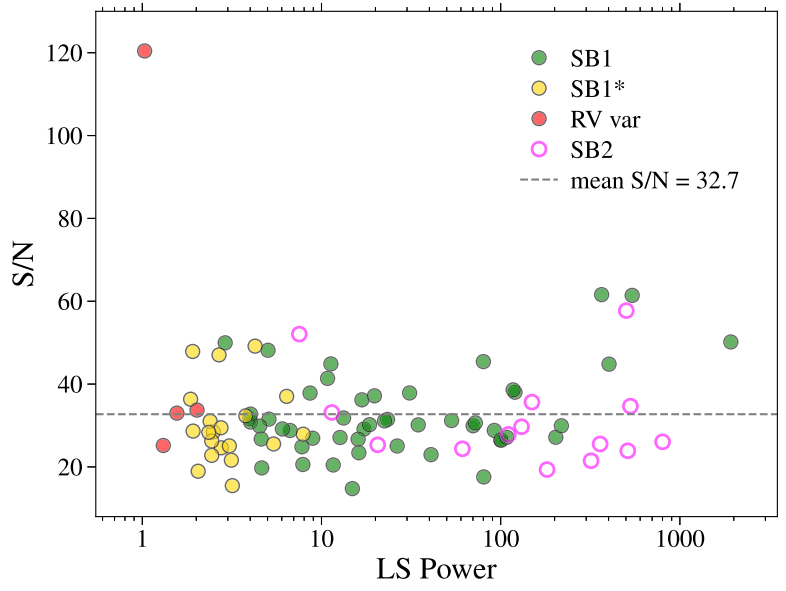

Figure 19. Correlation between $\mathrm{S} / \mathrm{N}$ of our targets and LS power.

our results. Fig. 19 shows the $\mathrm{S} / \mathrm{N}$ values for the BBC targets against the LS power of the maximum peak in the periodograms. There is no strong correlation between these values, although SB1 systems with the highest $\mathrm{S} / \mathrm{N}$ have also the highest LS power. It appears that $\mathrm{S} / \mathrm{N}$ did not play a significant role in the determination of orbital periods for most of our sample, but it remains as a possible source of uncertainty in the periods of systems with high $v_{e} \sin i$ and low semi-amplitude velocities, and it is still crucial in identifying SB2 systems with fainter secondaries.

\subsubsection{Be stars and non-radial pulsations}

Most Be stars are thought to present non-radial pulsations (NRPs), with periods of 0.5 to $2 \mathrm{~d}$ and amplitudes of $\lesssim 20 \mathrm{~km} \mathrm{~s}^{-1}$ (Rivinius, Carciofi \& Martayan 2013). If some of our period determinations are affected by NRP, this would be expected to happen for the shortperiod and low- $K$ systems.

In this context, we examined the semi-amplitude velocities and periods for the BBC sample. As expected, the short-period systems generally have higher $K$-values, especially the SB2 systems (see
Fig. 20). When we consider the SB1 and SB1* systems there are a handful of systems with $P_{\text {orb }}<2 \mathrm{~d}$ and $K_{1}<30 \mathrm{~km} \mathrm{~s}^{-1}$ (lower left in Fig. 20). These are two SB1 (VFTS 179 and 324) and four SB1* systems (VFTS 662, 697, 730, and 847). VFTS 697 and 847 are both Be stars, and VFTS 730 has a large rotational velocity $\left(v_{e} \sin i=248 \mathrm{~km} \mathrm{~s}^{-1}\right.$ ). Of the two SB1 systems, VFTS 324 has the most robust period and a well-constrained orbital solution, whereas VFTS 179 shows evidence of a possible alternative period of $\sim 7 \mathrm{~d}$ (see individual notes in Appendix A). Except for VFTS 324, each of these systems could be displaying periodicity from NRP. These results are particularly interesting for the Be stars as there is lack of known Be stars with MS companions. If not due to NRP, the low $K$-velocities of the Be stars would require an MS secondary or a compact companion. We note that a more detailed study of the Be stars from the VFTS (including those discussed here) is underway (Dufton et al., in preparation), including revisiting the estimates of $v_{e} \sin i$.

\subsection{Eclipsing binaries}

11 of our systems (including seven of the SB2s) were reported as EBs from analysis of the OGLE imaging survey (Pawlak et al. 2016). For 10 of the 11 systems, the OGLE and BBC periods show excellent agreement, with absolute differences ranging from 0.0042 to $0.000014 \mathrm{~d}$ ( $6 \mathrm{~min}$ to $1.2 \mathrm{~s}$ ). Our periods for VFTS 112 and 189 are also in excellent agreement with results using photometry from the EROS survey (Muraveva et al. 2014).

The only exception compared to the OGLE results is the SB2 system VFTS 883, where the published period is 2.00014 times our estimate. This can happen due to a mis-identification of the components. In fact, VFTS 883 has the lowest S/N ( 20) of the SB2 systems, affecting the number of epochs used in our LS analysis and, apparently, the correct identification of the components of the binary.

To search for such effects in our other SB2 systems, we conducted the LS analysis on the absolute difference between RVs of the two components in the SB2 systems. This test should produce signals that are half the orbital period (Almeida et al. 2017). Beyond the seven EBs, we confirmed the period estimates of four of our SB2 systems, whereas we did not find significant signals for the three remaining systems (VFTS 520, 589, 686). The latter two of these are the more eccentric systems, which probably limited this additional test, although given the good agreement between the overlapping systems in BBC and OGLE, our periods for these appear robust.

\subsection{Searching for compact companions}

The $K-P$ diagram in Fig. 20 is an interesting way of examining the sample because SB1 systems with a high $K_{1}$ are potential candidates to have compact companions. It is worth mentioning that these are semi-amplitudes of the projected orbital velocities meaning that we only see a fraction of the true velocity.

Two recent studies claimed to have found $\mathrm{BHs}$ in binary/multiple systems (Liu et al. 2019; Rivinius et al. 2020), but subsequent studies have proposed a different configuration involving a primary helium star and a Be secondary in both cases (Bodensteiner et al. 2020; Shenar et al. 2020). We have included these two objects, LB-1 and HR 6819, in Fig. 20 with values from Shenar et al. (2020) and Bodensteiner et al. (2020), respectively. We also include a third, shorter period system of interest in this context, NGC 2004\#115 (Lennon et al., in preparation, from the FLAMES data of Evans et al. 2006). These three systems each have $K_{1}>50 \mathrm{~km} \mathrm{~s}^{-1}$. We have 




Figure 20. Semi-amplitude velocity $\left(K_{1}\right)$ versus period $(P)$ for the BBC sample, compared with results for known and candidate $\mathrm{BH}$ systems from the literature. The red-dashed line represents systems with $M_{1}=15 \mathrm{M}_{\odot}, q$ $=1$, and $i=\pi / 6$, whereas the blue-solid line corresponds to $M_{1}=15 \mathrm{M}_{\odot}$, $q=0.7$, and $i=\pi / 4$. Shaded blue and red areas indicate the most probable regions for a $\mathrm{BH}$ companion from Langer et al. (2020), see text for details. The dashed line indicates the $8 \mathrm{~km} \mathrm{~s}^{-1}$ threshold $\left(\Delta \mathrm{RV}_{\min }=16 \mathrm{~km} \mathrm{~s}^{-1}\right)$ chosen by Dunstall et al. (2015).

also plotted in Fig. 20 four of the known OB-type stars with $\mathrm{BH}$ companions (Orosz et al. 2007, 2009, 2011; Casares et al. 2014). ${ }^{3}$ The known $\mathrm{BH}$ companions and recent candidates tend to have large $K_{1}$ velocities relative to the bulk of the $\mathrm{BBC}$ results for a given period. Several of the $\mathrm{BBC}$ systems are found in similar locations in the figure, with five SB1 systems with $K_{1}>70 \mathrm{~km} \mathrm{~s}^{-1}$. Additional data for these systems are required to see if they are simply outliers to the distribution or if they might also harbour $\mathrm{BH}$ companions.

Langer et al. (2020) recently suggested that about 1.5 per cent of the B-type stars in the LMC with masses above $10 \mathrm{M}_{\odot}$ could have $\mathrm{BH}$ companions, which would translate to about $60 \mathrm{~B}+\mathrm{BH}$ systems. Given the current population of HMXBs in the LMC (van Jaarsveld et al. 2018), binaries with compact companions are probably Xray quiet, as previously discussed by Casares et al. (2014). Langer et al. also predicted the distributions of periods and semi-amplitudes (their fig. 12) for systems resulting from Case A and B mass-transfer scenarios. For the Case A systems, they estimated the maximum probability of finding a $\mathrm{BH}$ companion for a given system at just above 2 per cent at a peak of $6.3 \mathrm{~d}$ in the orbital period distribution, and a probability of $\sim 1.5$ per cent at the peak of the semi-amplitude velocities $\left(130 \mathrm{~km} \mathrm{~s}^{-1}\right)$. The probabilities for the Case B systems are higher, with almost 8 per cent at a peak of $178 \mathrm{~d}$, and $>4.5$ per cent for peak velocities of $40 \mathrm{~km} \mathrm{~s}^{-1}$. This implies that most of the OB binaries with $\mathrm{BH}$ companions are expected to come from Case $\mathrm{B}$ evolution and, in fact, the period distribution of their Case B systems agrees well with that of Galactic Be/X-ray binaries.

The maximum probabilities, the peak in the distributions from fig. 12 in Langer et al. (2020), are included in our $K-P$ diagram (Fig. 20) as small blue (Case A, $P_{\text {orb }}=6.3 \mathrm{~d}, K_{1}=102.7 \mathrm{~km} \mathrm{~s}^{-1}$ ) and small red (Case B, $P_{\text {orb }}=177.8 \mathrm{~d}, K_{1}=31.6 \mathrm{~km} \mathrm{~s}^{-1}$ ) squares,

${ }^{3} \mathrm{M} 33-\mathrm{X} 7$ is a $70 \mathrm{M}_{\odot}$ O6 III with a $15.6 \mathrm{M}_{\odot} \mathrm{BH}$ companion (Pietsch et al. 2006; Orosz et al. 2007). LMC X-1 contains a $31.8 \mathrm{M}_{\odot}$ O8(f)p primary with a $10.91 \mathrm{M}_{\odot} \mathrm{BH}$ (Orosz et al. 2009; Walborn et al. 2010). Cyg X-1 is a 19.2 $\mathrm{M}_{\odot}$ O9.7 Iabpvar star with a $14.8 \mathrm{M}_{\odot} \mathrm{BH}$ secondary (Orosz et al. 2011; Sota et al. 2011). Finally, MWC 656 is the only known Be star (B1.5 IIIe, $M=10-16 \mathrm{M}_{\odot}$ ) with a BH companion of 3.8-6.9 $\mathrm{M}_{\odot}$ (Casares et al. 2014). reduced by 21 per cent for $K$ as suggested by Langer et al. due to projection effects. The larger rectangles in the figure indicate probabilities between $0-1$ per cent (largest blue rectangle) and $>1$ per cent (middle blue rectangle) for both $P$ and $K_{1}$ in the Case A systems, and probabilities between 1-3 per cent and 0-3 per cent (largest red rectangle) for $P$ and $K_{1}$, respectively, and $>3$ per cent (middle red rectangle) for both $P$ and $K_{1}$ for the Case B systems. These predicted regions match the location in the diagram of the known BH companions to OB-type stars and the other candidates; $33 \mathrm{BBC}$ systems are also within those regions, with 16 located in the higher probability region for the case B systems.

Curves for two different sets of parameters are plotted in Fig. 20 to help guide the discussion. The red curve indicates the position of systems with $M_{1}=15 \mathrm{M}_{\odot}, q=1$, and $i=\pi / 3$, effectively giving an upper boundary for the SB2 systems. The blue line was plotted for $M_{1}=15 \mathrm{M}_{\odot}, q=0.7$, and $i=\pi / 4$; this is a more typical value for $i$, but $q=0.7$ is close to the limit at which we can detect SB2 systems (albeit the curve here is for circular orbits, and the limit depends on $e$ as well as $P_{\text {orb }}$ ). None the less, for a flat $q$ distribution we expect the area below the curve for a sample such as ours to be mostly populated by SB1 systems, as observed in Fig. 20. Another reason to choose $q=0.7$ is that it was the average value at $\sim 15 \quad \mathrm{M}_{\odot}$ predicted by Langer et al. for $\mathrm{B}+\mathrm{BH}$ systems, with a rather narrow $q$-distribution. We therefore expect $\mathrm{B}+\mathrm{BH}$ binaries to be close to this line, which also passes through the regions of highest probability from the simulations. Furthermore, the locations of known and candidate BHs are well traced by the curve, with several $\mathrm{BBC}$ binaries close to the curve and four systems above it. The analysis of massive binaries through the $K-P$ diagram and the predictions from Langer et al. (2020) open interesting possibilities for future studies of this and other samples of massive stars. All BBC candidates to $\mathrm{B}+\mathrm{BH}$ systems will be investigated in more detail by Villaseñor et al. (in preparation).

\section{SUMMARY}

We have presented comprehensive multi-epoch spectroscopy of 88 candidate B-type binaries in the 30 Dor region of the LMC, analysed in the framework of the BBC programme. Using profile fits of (up to) nine absorption lines in the FLAMES spectra, we have estimated stellar RVs for each target for each epoch, and used LS tests to search these for periodicities from binary motion. For those with robust periods we estimated orbital properties (periods, eccentricity, mass ratios for the SB2 systems) using the RVFIT code, and then compare these with published distributions for more massive O-type stars in the Galaxy and LMC. Our findings include:

(i) Robust period estimates for $50 \mathrm{SB} 1$ and $14 \mathrm{SB} 2$ systems ranging between 1.16 and $428 \mathrm{~d}$, which comprises the largest homogeneous study of binary B-type stars to date. We have less secure periods for a further 20 systems (classified here as ' $S B 1^{*}$ '), with only four targets where our period search did not find a robust value.

(ii) Determination of full orbital solutions, including eccentricities and mass ratios for SB2 systems. The observed distributions show a preference for low eccentricities $(e<0.4)$ and mass ratios close to one, although observational biases could have an important effect, particularly for the latter.

(iii) The period distributions of the earliest B-type dwarfs (B00.7 types) compared to the dwarfs with later types (B1-2.5) are qualitatively quite different. Our statistical tests show there is only a $\sim 10$ per cent chance of these being drawn from the same parent distribution. Although of weak significance, the later-type 
objects have relatively few systems with longer-periods (>10-20 d). Whether this is an observational bias or is physically meaningful is not clear at present.

(iv) The period distribution from the $\mathrm{BBC}$ results shows no significant differences to those for O-type stars in the Galaxy (Sana et al. 2012), in 30 Dor (Almeida et al. 2017), nor the combined Galactic OB sample in Cyg OB2 (Kobulnicky et al. 2014). Of particular note, the period distribution of the early-type (B0-0.7) $\mathrm{BBC}$ results is in excellent agreement with that for O-type stars in 30 Dor (Almeida et al. 2017).

(v) We have investigated the semi-amplitude velocities $\left(K_{1}\right)$ of our confirmed binaries as a function of their orbital periods $\left(P_{\text {orb }}\right)$ in the $K-P$ diagram (see Fig. 20), compared to those of known/candidate $\mathrm{BH}$-binaries and theoretical predictions. We have identified several targets that have moderately large $K_{1}$ velocities that merit further study to investigate the nature of their companions (Villaseñor et al., in preparation).

In summary, our study suggests that the general properties of binaries in the early B-type domain are largely similar to those for their more massive O-type cousins, at both Galactic and LMC metallicities. This apparent invariance with mass and metallicity for massive stars is an important result for population-synthesis models that include binary evolution in the context of the rates of CCSNe and compact remnants. However, detailed modelling of the different observational biases affecting the $\mathrm{BBC}$ sample will need to be computed to determine the universality of our findings.

\section{ACKNOWLEDGEMENTS}

Based on observations at the European Southern Observatory Very Large Telescope in programme 096.D-0825. We thank Liz Bartlett for helpful discussions regarding the period analysis of these data, and Danny Lennon for important suggestions in the determination of orbital solutions. We are grateful to the referee for their thoughtful and constructive comments. JIV acknowledges funding from the National Agency for Research and Development (ANID) Scholarship Programme 'Doctorado en el extranjero' Grant No. 72170619. OHRA acknowledges funding from the European Union's Horizon 2020 research and innovation programme under the Marie Sklodowska-Curie grant agreement No. 665593 awarded to the Science and Technology Facilities Council. SdM was funded in part by the European Union's Horizon 2020 research and innovation programme from the European Research Council (ERC, grant agreement No. 715063), and by the Netherlands Organization for Scientific Research (NWO) as part of the Vidi research programme BinWaves with project number 639.042.728 and the National Science Foundation under NSF grant number 2009131.

\section{DATA AVAILABILITY}

Reduced and normalized data available on request.

\section{REFERENCES}

Abbott B. P. et al., 2017b, ApJ, 848, L13

Abbott B. P., Abbott R., Abbott T. D., Acernese F., Ackley K., Adams C., 2017a, Phys. Rev. Lett., 119, 161101

Abt H. A., Gomez A. E., Levy S. G., 1990, ApJS, 74, 551

Abt H. A., Levy S. G., 1978, ApJS, 36, 241

Almeida L. A. et al., 2017, A\&A, 598, A84

Astropy Collaboration, 2013, A\&A, 558, A33

Berger E., 2014, ARA\&A, 52, 43
Bodensteiner J. et al., 2020, A\&A, 641, A43

Casares J., Negueruela I., Ribó M., Ribas I., Paredes J. M., Herrero A., Simón-Díaz S., 2014, Nature, 505, 378

Chini R., Hoffmeister V. H., Nasseri A., Stahl O., Zinnecker H., 2012, MNRAS, 424, 1925

Clark J. S. et al., 2015, A\&A, 579, A131

Clark J. S., Tarasov A. E., Panko E. A., 2003, A\&A, 403, 239

Coulter D. A. et al., 2017, Science, 358, 1556

Crowther P. A. et al., 2016, MNRAS, 458, 624

Crowther P. A., Schnurr O., Hirschi R., Yusof N., Parker R. J., Goodwin S. P., Kassim H. A., 2010, MNRAS, 408, 731

Dai Z. G., Wang X. Y., Wu X. F., Zhang B., 2006, Science, 311, 1127

Doazan V. et al., 1986, A\&A, 158, 1

Duchêne G., Kraus A., 2013, ARA\&A, 51, 269

Dufton P. L. et al., 2011, ApJ, 743, L22

Dufton P. L. et al., 2013, A\&A, 550, A109

Duncan R. C., Thompson C., 1992, ApJ, 392, L9

Dunstall P. R. et al., 2012, A\&A, 542, A50

Dunstall P. R. et al., 2015, A\&A, 580, A93

Evans C. J. et al., 2011, A\&A, 530, A108

Evans C. J. et al., 2015, A\&A, 574, A13

Evans C. J., Lennon D. J., Smartt S. J., Trundle C., 2006, A\&A, 456, 623

Flannery B. P., van den Heuvel E. P. J., 1975, A\&A, 39, 61

Fong W., Berger E., 2013, ApJ, 776, 18

Garland R. et al., 2017, A\&A, 603, A91

Grin N. J. et al., 2017, A\&A, 600, A82

Hampel F. R., 1974, J. Am. Stat. Assoc., 69, 383

Hulse R. A., Taylor J. H., 1975, ApJ, 195, L51

Iglesias-Marzoa R., López-Morales M., Jesús Arévalo Morales M., 2015, PASP, 127, 567

Ingber L., 2000, preprint (cs/0001018)

Kiminki D. C., Kobulnicky H. A., 2012, ApJ, 751, 4

Kobulnicky H. A. et al., 2014, ApJS, 213, 34

Kobulnicky H. A., Fryer C. L., 2007, ApJ, 670, 747

Kramida A., Ralchenko Yu., Reader J., NIST ASD Team, 2020, NIST Atomic Spectra Database (version 5.8), https://doi.org/10.18434/T4W30F

Kroupa P., 2001, MNRAS, 322, 231

Langer N. et al., 2020, A\&A, 638, A39

Levato H., Malaroda S., Morrell N., Solivella G., 1987, ApJS, 64, 487

Liu J. et al., 2019, Nature, 575, 618

Lomb N. R., 1976, Ap\&SS, 39, 447

Martins F., Schaerer D., Hillier D. J., 2005, A\&A, 436, 1049

Mason B. D., Hartkopf W. I., Gies D. R., Henry T. J., Helsel J. W., 2009, AJ, 137,3358

McEvoy C. M. et al., 2015, A\&A, 575, A70

Moe M., Di Stefano R., 2013, ApJ, 778, 95

Moe M., Di Stefano R., 2015, ApJ, 810, 61

Moe M., Di Stefano R., 2017, ApJS, 230, 15

Muraveva T. et al., 2014, MNRAS, 443, 432

Newville M., Stensitzki T., Allen D. B., Ingargiola A., 2014, LMFIT: NonLinear Least-Square Minimization and Curve-Fitting for Python, https: //doi.org/10.5281/ zenodo.11813

Orosz J. A. et al., 2007, Nature, 449, 872

Orosz J. A. et al., 2009, ApJ, 697, 573

Orosz J. A., McClintock J. E., Aufdenberg J. P., Remillard R. A., Reid M. J., Narayan R., Gou L., 2011, ApJ, 742, 84

Pawlak M. et al., 2016, Acta Astron., 66, 421

Pietsch W., Haberl F., Sasaki M., Gaetz T. J., Plucinsky P. P., Ghavamian P., Long K. S., Pannuti T. G., 2006, ApJ, 646, 420

Price-Whelan A. M. et al., 2018, AJ, 156, 123

Raboud D., 1996, A\&A, 315, 384

Ramírez-Agudelo O. H. et al., 2013, A\&A, 560, A29

Reig P., 2011, Ap\&SS, 332, 1

Rivinius T., Baade D., Hadrava P., Heida M., Klement R., 2020, A\&A, 637, L3

Rivinius T., Carciofi A. C., Martayan C., 2013, A\&AR, 21, 69

Rousseeuw P. J., Croux C., 1993, J. Am. Stat. Assoc., 88, 1273 
Rowlinson A., O’Brien P. T., Metzger B. D., Tanvir N. R., Levan A. J., 2013, MNRAS, 430, 1061

Sana H. et al., 2012, Science, 337, 444

Sana H. et al., 2013, A\&A, 550, A107

Sana H. et al., 2014, ApJS, 215, 15

Scargle J. D., 1982, ApJ, 263, 835

Shenar T. et al., 2020, A\&A, 639, L6

Smartt S. J. et al., 2017, Nature, 551, 75

Sota A., Maíz Apellániz J., Walborn N. R., Alfaro E. J., Barbá R. H., Morrell N. I., Gamen R. C., Arias J. I., 2011, ApJS, 193, 24

Stankov A., Handler G., 2005, ApJS, 158, 193

Tauris T. M. et al., 2017, ApJ, 846, 170

van Jaarsveld N., Buckley D. A. H., McBride V. A., Haberl F., Vasilopoulos

G., Maitra C., Udalski A., Miszalski B., 2018, MNRAS, 475, 3253

VanderPlas J. T., 2018, ApJS, 236, 16

Waelkens C., Aerts C., Kestens E., Grenon M., Eyer L., 1998, A\&A, 330, 215

Walborn N. R. et al., 2010, AJ, 139, 1283

Walborn N. R. et al., 2014, A\&A, 564, A40

Wolff S. C., 1978, ApJ, 222, 556

\section{SUPPORTING INFORMATION}

Supplementary data are available at MNRAS online.

Figure S1 Periodograms of the SB1 systems.

Figure S2 Periodograms of the SB1* systems (possible periods).

Figure S3 Periodograms of the SB2 systems.

Figure S4 Radial velocity curves of the SB1 systems.

Figure S5 Radial velocity curves of the SB1* systems (possible periods).

Figure S6 Radial velocity curves of the SB2 systems.

Please note: Oxford University Press is not responsible for the content or functionality of any supporting materials supplied by the authors. Any queries (other than missing material) should be directed to the corresponding author for the article.

\section{APPENDIX A: NOTES ON INDIVIDUAL OBJECTS}

The following notes describe difficulties we had finding periods for specific systems (e.g. particularities found in the spectra and/or in the periodograms of some of our targets) and further details on the tests conducted, specifically our lines combination test (LCT) described in Section 3.2.3.

VFTS 018: Possible period at $70.8 \mathrm{~d}$ but the peak does not surpass the 0.1 per cent FAP. The LCT analysis found 46 per cent of the line combinations with the peak at $70.8 \mathrm{~d}$, but also found peaks at $3.9 \mathrm{~d}$ (16 per cent of line sets) and $61 \mathrm{~d}$ (6 per cent).

VFTS 037: Weak nebular emission is present in all the helium lines and we carried out the analysis with and without fitting the nebular emission. Two peaks were detected at 41 and $71 \mathrm{~d}$ using different sets of lines, with the latter period slightly favoured by fits without nebular emission. We adopted $P_{\text {orb }}=41 \mathrm{~d}$ as its peak is above the 0.1 per cent FAP, but flag it as only a 'possible' period given the other peak. Our LCT for fits including nebular emission recovered $P_{\text {orb }}=41 \mathrm{~d}$ for 20 per cent of the possible line sets (in which all included a larger peak at $1 \mathrm{~d}$, most likely an alias), and where the peak at $41 \mathrm{~d}$ was above the 0.1 per cent FAP for two combinations. The possible period of $71 \mathrm{~d}$ was only recovered for 12 per cent of the line sets, where it neither surpassed the 0.1 per cent FAP nor the strength of the alias close to $1 \mathrm{~d}$. A third peak at $46 \mathrm{~d}$ was also present in the periodogram and presented a feasible solution with RVFIT, but its peak in the periodogram was always weaker than that at $41 \mathrm{~d}$.

VFTS 097: Although the RV curve looks noisy and its peak in the periodogram is slightly above the 0.1 per cent FAL, we confirmed the estimated $19.8 \mathrm{~d}$ period for 55 per cent of line combinations from the LCT.

VFTS 106: Possible period of 16.4 d found for 24 per cent of the different sets of lines, all of which included He I $\lambda 4471$ (which presents weak nebular emission). Its RV data are well fit by RVFIT with one of the lowest $\chi^{2}$ values among the SB1* systems.

VFTS 144: Two peaks are present at $P_{\text {orb }} \sim 171$ and $\sim 351 \mathrm{~d}$ but neither surpasses the 1 per cent FAP threshold.

VFTS 146: The LCT found $P_{\text {orb }}=117 \mathrm{~d}$ (that we missed in our initial analysis) for 52 per cent of the line sets. Given the weak signal in periodogram and resulting RV curve, we have therefore classified this system as $\mathrm{SB} 1^{*}$.

VFTS 155: Two peaks at $\sim 154$ and $\sim 335 \mathrm{~d}$, but $P_{\text {orb }} \sim 154 \mathrm{~d}$ is adopted as it exceeds the 1 per cent FAP in 12 per cent of the cases when running the LCT. We have included this system in the SB1* group.

VFTS 162: The spectra indicate line-profile variability or a very weak secondary. Periods of 98 and $144 \mathrm{~d}$ were estimated from different sets of lines, with the longer period the most common and prominent. The longer period presents the clearer periodogram with the stronger peak and was adopted as the orbital period of this system.

VFTS 179: The shortest period binary in our sample with $P_{\text {orb }}=1.16 \mathrm{~d}$. A second, weaker peak is present in the periodogram at $7 \mathrm{~d}$, but the shorter period had the maximum peak in the periodogram for 62 per cent of the line combinations and is adopted as the period. However, we note that we found a plausible orbital solution for the $7 \mathrm{~d}$ period, with $e=0.28, K_{1}=18.9 \mathrm{~km} \mathrm{~s}^{-1}$, and $\chi_{\text {red }}^{2}=1.15$, but it relies on $\mathrm{RVs}$ from the weak $\mathrm{Mg}$ II.

VFTS 204: We found $P_{\text {orb }}=157 \mathrm{~d}$ for this system only after checking with our LCT. Although we found some scatter, with periods between 148 and $161 \mathrm{~d}$, the $157 \mathrm{~d}$ period was recovered for 36 per cent of the possible line combinations. The RVFIT analysis computed a final period of $164 \mathrm{~d}$, with the highest eccentricity value among the SB1 systems.

VFTS 206: Given the observed profile in the stronger He I $\lambda 4026$ and $\lambda 4471$ lines, it is possible that the primary is a fast rotator with a B8 secondary. In addition to the solution reported in Table B6, we also investigated the scenario of a potentially smaller mass ratio. The signal remains very strong for the same period $(7.56 \mathrm{~d})$, and we found an orbital solution with $q=0.19$. For a typical B8 mass of 3.5 $\mathrm{M}_{\odot}$, this implies $M_{1} \sim 18 \mathrm{M}_{\odot}$. However, knowing the limitations of our observational campaign to detect such mass ratios, we favoured the solution with more similar masses.

VFTS 213: A possible period of $13.5 \mathrm{~d}$ was determined for this Be star from a peak above 1 per cent FAP present for 57 per cent of the possible sets of lines. However, two stronger peaks at 0.5 and $1 \mathrm{~d}$ are present in the periodograms of all the cases found with the LCT, which might be aliases of the longer period. Its RV curve presents significant scatter and appears to have a high eccentricity.

VFTS 218: Presents line-profile variability in its helium lines, most noticeably in $\mathrm{He}$ I $\lambda \lambda 4388,4471$. The variability shifts between the red and blue wing which might be caused by a weak secondary. A possible period of $20.8 \mathrm{~d}$ was found from a weak signal above the 1 per cent FAP, which was also found in 19 per cent of the cases with our LCT, and for an additional 55 per cent as a secondary peak. A weaker peak is also present in the periodogram at $80 \mathrm{~d}$ that does 
not improve the orbital solution. As in the case of VFTS 213, its RV curve presents significant scatter, high eccentricity, and a high $\chi^{2}$ value.

VFTS 240: A SB2 system with a weak secondary component only visible in a few epochs that makes it difficult to fit. We have confirmed its $1.38 \mathrm{~d}$ period by fitting it also as a SB1 and including the hydrogen lines.

VFTS 255: This SB2 system presents nebular emission in He I $\lambda 4026$ and $\lambda 4471$. We confirmed its period by both using and excluding these lines from the RV measurements to determine the period.

VFTS 257: Although its RV curve presents some scatter, 71 per cent of line combinations indicated $P_{\text {orb }}=132 \mathrm{~d}$.

VFTS 291: Presents some line-profile variability in He I $\lambda 4026$ that looks to be mild nebular emission. This does not appear to be the case from inspection of He I $\lambda 4471$, which could suggest signs of a weak secondary. It has a $107.6 \mathrm{~d}$ period with a circular orbit.

VFTS 299: Another system displaying line-profile variability. We are unable to determine if its variability is due to weak nebular emission or a secondary. Its period of $20.3 \mathrm{~d}$ was recovered for 100 per cent of the different sets of lines with our LCT, although the RV curve presents some scatter and high eccentricity.

VFTS 337: One of our six Be stars. It presents emission in all the spectral lines available for RV measurements and the weak helium lines are probably also broadened by rotation. We were initially unable to find a period for this system but further checks with our LCT showed a peak at $27 \mathrm{~d}$ for 12 per cent of the possible lines sets, which we have included as a possible period.

VFTS 359: Line-profile variability is noticeable in the wings of $\mathrm{He}$ I $\lambda 4388$, especially for epochs 6,21 , and 23 . It is unclear if these features are from lunar contamination (i.e. linked to the background subtraction), have a nebular origin or hint at a faint secondary. A peak at $19.5 \mathrm{~d}$ is present for 67 per cent of the possible lines combinations confirming our initial period.

VFTS 364: As with VFTS 255, we have confirmed the period of this SB2 system with and without the use of He I $\lambda 4026$ and $\lambda 4471$ that present clear nebular contamination.

VFTS 383: We have confirmed the SB2 status of this binary due to the presence of Si III $\lambda 4553$ from the secondary spectrum, which was otherwise difficult to determine given the nebular contamination in the He I $\lambda 4026$ and $\lambda 4471$ lines.

VFTS 388: Three different peaks were recovered using the LCT at $14.8,20.4$, and $61.7 \mathrm{~d}$ for line sets of 24,2 , and 31 per cent, respectively. In most of these cases, it was a secondary peak, smaller than the 0.5 and $1 \mathrm{~d}$ aliases. We adopt $P_{\text {orb }}=14.8 \mathrm{~d}$ as the tentative period for the system since it has the strongest peak and is the only case with a primary peak.

VFTS 391: Nebular emission was present in the available Balmer and helium lines, whereas the metallic lines were too weak to be fitted. We did not find signs of periodicity.

VFTS 396: Subtle nebular emission in He I $\lambda 4026$ and $\lambda 4388$. The $85 \mathrm{~d}$ period was found for 48 per cent of the different sets of lines and seems robust from the periodogram with a peak above the 0.1 per cent FAL. We have found some dispersion in the period with our LCT between 83.7 and $92.1 \mathrm{~d}$, similar to the case of VFTS 204, and a final period of $86 \mathrm{~d}$ with RVFIT.

VFTS 442: Possible period at $27.1 \mathrm{~d}$ revealed by our LCT for 21 per cent of the possible line sets. While the peak in the periodogram is clear, the RVs present larger errors and scatter in comparison to the semi-amplitude velocity in the blueshifted (lower) portion of the RV curve.
VFTS 501: The spectra show evidence that this could be a SB2 system. However, strong nebular emission in He I $\lambda 4026$ and $\lambda 4471$ suggests that the apparent secondary component seen in the weaker He lines ( $\lambda 4144$ and $\lambda 4388)$ might be an artefact of weaker nebular emission. We measured the centre of the emission and determined that it is moving with a maximum difference of $0.8 \AA$ in $\mathrm{He} \mathrm{I} \lambda 4026$. We analysed the system as a SB2 finding a period of $99 \mathrm{~d}$ with unrealistic minimum masses of 127 and $59 \mathrm{M}_{\odot}$, so we decided to treat it as a SB1, for which we determined a period of 151d.

VFTS 575: The $46 \mathrm{~d}$ period was recovered for 99 per cent of the possible line combinations indicating a robust period. Regardless of the low LS power in the periodogram $\left(P_{\text {peak }}<3\right)$, the RV data are remarkably well fitted by the solution found with RVFIT.

VFTS 576: Two similar peaks above 0.1 per cent FAL are present in the periodogram at 115.8 and $85.2 \mathrm{~d}$. The Balmer lines are affected by broad nebular emission, interfering with the fit. Our LCT was inconclusive, so we are not able to securely determine its period nor explain the presence of a second peak, so this system was classified as $\mathrm{SB} 1 *$. Nonetheless, we adopt the $85 \mathrm{~d}$ period as it presents a far better orbital solution from comparison of RV curves. VFTS 576 presents a high semi-amplitude velocity compared with other systems with similar periods and a large mass function.

VFTS 591: This is the brightest target (B0.2 Ia) and its spectra have the highest $\mathrm{S} / \mathrm{N}$ in our sample. A period of $468 \mathrm{~d}$ is generally present in the periodogram for different sets of lines, but the more significant peaks (above 1 per cent FAL) arise from including the Balmer lines, which suffer slight nebular contamination (which is challenging to fit). Given the high $\mathrm{S} / \mathrm{N}$ ratio of the data, we can estimate accurate RVs without including the Balmer lines, but we were unable to obtain a robust period estimate. Moreover, inclusion of the original VFTS spectra did not improve the signal in the periodogram (at which point the peak close to $500 \mathrm{~d}$ reported in Table B4 disappeared). Its $\mathrm{RV}$ variability might come from a non-binary origin.

VFTS 606: Besides the peak at $86.6 \mathrm{~d}$, there was a slightly stronger alias at $1 \mathrm{~d}$ and a third peak at $73 \mathrm{~d}$, which for 7 per cent of lines sets in our LCT was the primary peak. For these reasons, we have classified this system as SB1*.

VFTS 662: Presents very shallow helium lines and double-peaked emission in its Balmer lines. Classified as B3-5 III (Evans et al. 2015), it is one of the latest-type objects in our sample. We were unable to find a reliable period as its helium lines are broad and very weak. It is evident that He I $\lambda 4471$ is filled-in by double-peaked emission, which could also be the case for the other helium lines. Using Mg II $\lambda 4481$, we determined a possible period of $1.99 \mathrm{~d}$. We relaxed our condition of a minimum of three lines to two in our LCT for this system, and from the 120 possible sets, the $1.99 \mathrm{~d}$ period was only retrieved in 4 per cent of the cases. However, the LS peak is close to the 0.1 per cent FAL that gives some confidence in this period, although its RV curve presents some scatter and a low amplitude.

VFTS 665: Clear peak around $330 \mathrm{~d}$ but with considerable uncertainty between 302 and 366. However, 86 per cent of combinations from our LCT yielded a period in this range. We have determined a period of $324 \mathrm{~d}$ with RVFIT.

VFTS 686: Arguably the least obvious SB2 system in our sample. The second component is not resolved in the data and its presence is inferred from the shape of the wings of the helium lines of the giant primary. A peak at $16.9 \mathrm{~d}$ is present for every combination of lines arguing that this is a reliable period. Garland et al. (2017) also identified this system as a SB2 from the combined LR03 VFTS spectra.

VFTS 697: Presents strong nebular emission in its Balmer lines and He I $\lambda 4471$. Using only the three helium lines free (or less affected) 
from nebular contamination, it was possible to retrieve a short period of $1.49 \mathrm{~d}$. From our LCT, this period was found for only 7 per cent of the cases, but this might be due to the strong nebular contamination. Its RV curve displays considerable scatter and it was classified only as $\mathrm{SB} 1^{*}$.

VFTS 730: We found a short possible period of $1.33 \mathrm{~d}$ for only one combination of lines. We considered it as 'possible' given that the corresponding peak in the periodogram is close to the 0.1 per cent FAL. We note that this system has a considerably higher $\gamma$-velocity than the rest of the sample.

VFTS 784: We could not find signs of periodicity for this system. However, lowering our requirement for the minimum number of lines for RV determination to two, the LCT showed a peak above the 1 per cent FAP level at $3.6 \mathrm{~d}$, with a stronger alias at $0.8 \mathrm{~d}$. This period was only recovered for 5 per cent of the possible line sets and the periodogram presented was obtained using two lines $(\mathrm{H} \delta$ and $\mathrm{He} \mathrm{I}$ $\lambda 4388$ ), so we consider this only as a possible period and classified it as $\mathrm{SB} 1 *$.

VFTS 792: Only 14 epochs of observations were obtained for VFTS 792, from which two were unusable due to low S/N. Our LCT suggested an orbital period larger than $400 \mathrm{~d}$ but the spread in possible periods was large (from 460 to $940 \mathrm{~d}$ ) as a consequence of our limited sensitivity at such large periods and due to the small number of epochs for this particular system. Given its possible long period, we included the original observations taken by the VFTS to increase the number of epochs to 17 . We have also used He I $\lambda 4009$ and $\mathrm{Mg}$ II $\lambda 4481$ which were strong enough to be fitted in this case. We retrieved $P_{\text {orb }}=431.7 \mathrm{~d}$ with a clear and strong signal with our LS test and a final period of $428 \mathrm{~d}$ with RVFIT.

VFTS 799: After first tests, we expanded our set of spectral lines to include He I $\lambda 4009$ and $\mathrm{Mg}$ II $\lambda 4481$ and ran our LCT with a set of nine lines. Our test retrieved a peak at $39.4 \mathrm{~d}$ but only for 2 per cent of the possible combinations (eight sets) which are all related to the metallic Si III and Mg II lines. This is one of the most highly eccentric binaries in the SB1* group $(e=0.69)$ and with the lowest semi-amplitude velocity $\left(K_{1}=6.34 \mathrm{~km} \mathrm{~s}^{-1}\right)$.

VFTS 847: This was classified as B0.7-1 IIIne by Evans et al. (2015) but $\mathrm{Si}$ III $\lambda 4553$ is absent from the BBC data or indistinguishable from noise. Considering the rotational broadening, this seems to be an earlier-B MS star. A peak at $1.2 \mathrm{~d}$ was recovered for only 4 per cent of line combinations, with a secondary peak at $5.2 \mathrm{~d}$ that might be an alias of the former period or the true period. Given the small number of lines from which it was possible to determine a period, the presence of a strong alias and the significant scatter in the $\mathrm{RV}$ curve, we classified this system as SB1*.
VFTS 850: The LCT, including He I $\lambda 4009$ and $\mathrm{Mg}$ II $\lambda 4481$, for this initially 'aperiodic' system found a weak but persistent signal at $50.5 \mathrm{~d}$, usually accompanied by a secondary peak around $111 \mathrm{~d}$. The former peak was present in 43 per cent of possible line sets and was the primary peak in 5 per cent of the cases (23 different sets). However, given the low power of the LS peak and the large scatter in the RV curve, we have classified this system as $\mathrm{SB} 1^{*}$.

VFTS 874: A long period of $371 \mathrm{~d}$ was found for this system with RVFIT; however, a second peak around $190 \mathrm{~d}$ was also present in the periodogram. The shorter peak is probably an alias of the true period and presents a more noisy RV curve. The LCT showed that 80 per cent of combinations favoured the longer period, with a dispersion in the range of 360 and $407 \mathrm{~d}$.

VFTS 877: Spectra of this Be star present deep narrow cores in the Balmer lines and He I $\lambda 4471$ from epoch 1 to 22 , after which it disappears. This variability might be associated with the dynamics of the decretion disc (e.g. Doazan et al. 1986; Clark, Tarasov \& Panko 2003), but requires further investigation. Two periods around 83 and $93 \mathrm{~d}$ were recovered, with the latter being the most significant. Its LS periodogram shows a clear peak just above the 0.1 per cent FAL. However, given the issues regarding the Balmer and He I $\lambda 4471$ lines (and use of other lines did not yield any orbital period), we have only classified the system as $\mathrm{SB} 1^{*}$.

VFTS 883: For SB2 systems, we have applied the LS test to the difference between RV measurements of primary and secondary components as for the TMBM sample. This test should retrieve a period that is half the orbital period. VFTS 883 was the only case where we found the same period in both cases which is an indication that the true period could be twice our determined period. If this were the case, going from 3.5 to $7 \mathrm{~d}$ will not have any major impact in the orbital period distribution.

VFTS 890: Displays a strong, good S/N, helium spectrum, however, we were not able to determine a robust orbital period. A signal of $18.9 \mathrm{~d}$ was found with the LCT in 56 per cent of the possible line sets, but only as the primary peak in 16 per cent of all possible cases, and in none of these cases is the peak is above the 1 per cent FAP level. We report this system only as an RV variable.

\section{APPENDIX B：TABLES}


Table B1. Properties of the BBC sample. Spectral classifications, magnitudes, and colours are from Evans et al. (2015). Physical parameters, including projected rotational velocities $\left(v_{e} \sin i\right)$, are from Dufton et al. (2013) and Garland et al. (2017) for dwarfs (LC V-III) and from McEvoy et al. (2015) for the supergiants. SB classifications are from this work.

\begin{tabular}{|c|c|c|c|c|c|c|c|c|c|c|c|c|c|c|}
\hline VFTS & SpT & $\mathrm{LC}$ & SB & V & $B-V$ & $\begin{array}{c}v_{e} \sin i \\
\left(\mathrm{~km} \mathrm{~s}^{-1}\right)\end{array}$ & $\log L / \mathrm{L}_{\odot}$ & $\begin{array}{l}T_{\text {eff }} \\
(\mathrm{K})\end{array}$ & $\begin{array}{c}\log g \\
\left(\mathrm{~cm} \mathrm{~s}^{-2}\right)\end{array}$ & $\begin{array}{c}\mathrm{M}_{\mathrm{s}} \\
\left(\mathrm{M}_{\odot}\right)\end{array}$ & $\begin{array}{c}\mathrm{M}_{\mathrm{e}} \\
\left(\mathrm{M}_{\odot}\right)\end{array}$ & $\begin{array}{c}t \\
(\mathrm{Myr})\end{array}$ & $\alpha(2000)$ & $\delta(2000)$ \\
\hline 9 & B1-1.5 & V & SB1 & 16.18 & 0.11 & 87 & - & - & - & - & - & - & 053704.26 & -690805.65 \\
\hline 15 & B0.5 & $\mathrm{V}$ & SB1 & 16.20 & 0.04 & 185 & - & - & - & - & - & - & 053708.56 & -690510.03 \\
\hline 18 & B1.5 & V & $\mathrm{SB} 1^{*}$ & 16.62 & 0.17 & 48 & 4.20 & - & - & - & - & - & 053711.46 & -691030.97 \\
\hline 27 & B1 & III-II & SB1 & 14.71 & 0.09 & 91 & 4.63 & 20500 & 3.10 & 12 & 14 & 13.18 & 053717.37 & -690748.05 \\
\hline 33 & B1-1.5 & $\mathrm{V}$ & SB1 & 16.20 & 0.07 & 77 & 4.26 & 24000 & 3.90 & - & - & - & 053722.05 & -690755.13 \\
\hline 37 & B2 & III: & $\mathrm{SB} 1^{*}$ & 15.79 & 0.06 & 267 & - & - & - & - & - & - & 053723.95 & -691221.27 \\
\hline 106 & B 0.2 & $\mathrm{~V}$ & $\mathrm{SB} 1^{*}$ & 16.43 & 0.05 & 170 & - & - & - & - & - & - & 053740.22 & -690412.11 \\
\hline 112 & EarlyBs & - & SB2 & 16.19 & 0.08 & 408 & - & - & - & - & - & - & 053740.89 & -690441.52 \\
\hline 144 & B0.7: & V & RV var & 16.81 & 0.00 & 154 & - & - & - & - & - & - & 053746.08 & -690914.37 \\
\hline 146 & B2: & $\mathrm{V}$ & $\mathrm{SB} 1^{*}$ & 16.24 & 0.25 & 287 & - & - & - & - & - & - & 053746.10 & -690634.93 \\
\hline 155 & B0.7: & V & $\mathrm{SB} 1^{*}$ & 16.95 & 0.08 & 277 & - & - & - & - & - & - & 053747.18 & -691313.60 \\
\hline 157 & B2 & $\mathrm{V}$ & SB1 & 16.69 & -0.08 & 175 & - & - & - & - & - & - & 053747.23 & -685849.25 \\
\hline 199 & EarlyBs & - & SB2 & 16.91 & -0.01 & - & - & - & - & - & - & - & 053754.78 & -690024.99 \\
\hline 204 & B2 & III & SB1 & 16.13 & 0.31 & $\leq 40$ & 4.41 & 22500 & 3.50 & - & - & - & 053755.04 & -690702.20 \\
\hline 206 & B3 & III: & SB2 & 14.99 & 0.15 & $<40$ & - & - & - & - & - & - & 053755.43 & -685706.99 \\
\hline 211 & B1 & $\mathrm{V}$ & SB1 & 16.56 & -0.12 & 188 & - & - & - & - & - & - & 053757.34 & -685841.89 \\
\hline 213 & B2 & III:e & $\mathrm{SB} 1^{*}$ & 15.50 & 0.04 & 181 & - & - & - & - & - & - & 053757.96 & -690954.11 \\
\hline 215 & B1.5 & $\mathrm{V}$ & SB1 & 16.58 & -0.03 & 154 & - & - & - & - & - & - & 053758.03 & -690223.54 \\
\hline 218 & B1.5 & $\mathrm{V}$ & $\mathrm{SB} 1^{*}$ & 15.63 & 0.38 & 79 & 4.89 & - & - & - & - & - & 053759.71 & -691114.29 \\
\hline 225 & B $0.7-1$ & III-II & SB1 & 15.07 & -0.01 & $\leq 40$ & 4.53 & 24500 & 3.25 & - & - & - & 053800.96 & -685723.28 \\
\hline 227 & B2 & $\mathrm{V}$ & SB1 & 16.51 & -0.09 & 183 & - & - & - & - & - & - & 053801.31 & -690313.91 \\
\hline 240 & B1-2 & V & SB2 & 15.85 & 0.01 & 77 & - & - & - & - & - & - & 053806.75 & -690608.76 \\
\hline 246 & B1 & III & SB1 & 16.83 & 0.45 & 135 & - & - & - & - & - & - & 053808.86 & -691040.04 \\
\hline 248 & B2: & $\mathrm{V}$ & SB2 & 16.49 & 0.00 & 268 & - & - & - & - & - & - & 053809.32 & -691014.33 \\
\hline 337 & B2: & V-IIIe+ & $\mathrm{SB} 1^{*}$ & 16.72 & 0.14 & 412 & - & - & - & - & - & - & 053825.60 & -690604.31 \\
\hline 342 & B1 & V & SB1 & 16.94 & -0.04 & $\leq 40$ & 3.84 & - & - & - & - & - & 053826.86 & -690417.82 \\
\hline 351 & B 0.5 & $\mathrm{~V}$ & SB1 & 15.98 & 0.08 & $\leq 40$ & 4.47 & 28500 & 4.00 & - & - & - & 053828.39 & -690640.89 \\
\hline 359 & B 0.5 & $\mathrm{~V}$ & SB1 & 16.30 & 0.03 & 54 & 4.27 & 28000 & 4.00 & - & - & - & 053829.39 & -690559.19 \\
\hline 364 & B2.5: & V & SB2 & 16.82 & 0.03 & 140 & - & - & - & - & - & - & 053830.07 & -690827.60 \\
\hline 383 & B0.5: & V & SB2 & 16.10 & 0.20 & 164 & - & - & - & - & - & - & 053832.31 & -690732.22 \\
\hline 388 & B 0.5 & $\mathrm{~V}$ & $\mathrm{SB} 1^{*}$ & 16.42 & 0.06 & 98 & - & - & - & - & - & - & 053832.63 & -690355.93 \\
\hline 391 & B0.5: & V & RV var & 16.32 & -0.02 & 260 & - & - & - & - & - & - & 053832.80 & -690524.92 \\
\hline 396 & B0.5 & $\mathrm{V}$ & SB1 & 16.32 & 0.11 & 131 & - & - & - & - & - & - & 053833.27 & -691024.00 \\
\hline 430 & B 0.5 & $\mathrm{Ia}+((\mathrm{n})) \mathrm{Nwk}$ & SB1 & 15.11 & 0.64 & 98 & 5.56 & 24500 & 2.65 & 18 & - & - & 053836.87 & -690646.08 \\
\hline 434 & B1.5: & $\mathrm{V}$ & SB1 & 16.13 & 0.16 & 45 & 4.38 & - & - & - & - & - & 053837.22 & -690425.98 \\
\hline 442 & B1-2 & $\mathrm{V}$ & $\mathrm{SB} 1^{*}$ & 16.66 & 0.08 & 281 & - & - & - & - & - & - & 053837.89 & -690336.76 \\
\hline 501 & B 0.5 & V & SB1 & 15.74 & 0.08 & 59 & 4.57 & - & - & - & - & - & 053841.23 & -690414.22 \\
\hline 520 & B1: & V & SB2 & 16.69 & 0.08 & 53 & 4.11 & - & - & - & - & - & 053841.99 & -690653.46 \\
\hline 534 & B0 & IV & SB1 & 15.66 & 0.21 & 57 & 4.82 & 29000 & 3.75 & - & - & - & 053842.80 & -691540.29 \\
\hline 575 & B0.7 & III & SB1 & 15.11 & 0.02 & $\leq 40$ & 4.57 & 26000 & 3.75 & - & - & - & 053844.91 & -690533.10 \\
\hline 576 & $\mathrm{~B} 1$ & IaNwk & $\mathrm{SB} 1^{*}$ & 15.67 & 0.78 & 52 & 5.31 & 20000 & 2.50 & 16 & 32 & 4.90 & 053844.94 & -691511.45 \\
\hline 589 & B0.5 & V & SB2 & 15.83 & 0.15 & $\leq 40$ & 4.63 & 27500 & 4.00 & - & - & - & 053845.59 & -690734.86 \\
\hline 591 & B0.2 & Ia & RV var & 12.55 & 0.23 & 48 & 5.91 & 25000 & 2.80 & 53 & 48 & 3.31 & 053845.69 & -690622.45 \\
\hline 606 & B0-0.5 & $\mathrm{V}(\mathrm{n})$ & $\mathrm{SB} 1^{*}$ & 16.60 & 0.06 & 86 & - & - & - & - & - & - & 053846.71 & -690538.78 \\
\hline 637 & B1-2 & V+EarlyB & SB2 & 16.61 & 0.04 & - & - & - & - & - & - & - & 053849.40 & -690615.28 \\
\hline 662 & B3-5 & III: & $\mathrm{SB} 1^{*}$ & 16.12 & 0.08 & 67 & 3.94 & 17500 & 3.60 & - & - & - & 053852.55 & -690220.30 \\
\hline
\end{tabular}


Table B1 - continued

\begin{tabular}{|c|c|c|c|c|c|c|c|c|c|c|c|c|c|c|}
\hline VFTS & SpT & $\mathrm{LC}$ & SB & V & $B-V$ & $\begin{array}{c}v_{e} \sin i \\
\left(\mathrm{~km} \mathrm{~s}^{-1}\right)\end{array}$ & $\log L / \mathrm{L}_{\odot}$ & $\begin{array}{l}T_{\text {eff }} \\
(\mathrm{K})\end{array}$ & $\begin{array}{c}\log g \\
\left(\mathrm{~cm} \mathrm{~s}^{-2}\right)\end{array}$ & $\begin{array}{c}\mathrm{M}_{\mathrm{s}} \\
\left(\mathrm{M}_{\odot}\right)\end{array}$ & $\begin{array}{c}\mathrm{M}_{\mathrm{e}} \\
\left(\mathrm{M}_{\odot}\right)\end{array}$ & $\begin{array}{c}t \\
(\mathrm{Myr})\end{array}$ & $\alpha(2000)$ & $\delta(2000)$ \\
\hline 665 & B 0.5 & V & SB1 & 16.43 & 0.11 & 47 & 4.33 & 28000 & 4.15 & - & - & - & 053852.74 & -690728.71 \\
\hline 686 & В 0.7 & III & SB2 & 14.99 & 0.17 & $\leq 40$ & 4.83 & 24000 & 3.60 & - & - & - & 053855.63 & -690723.77 \\
\hline 687 & B1.5 & $\mathrm{Ib}((\mathrm{n})) \mathrm{Nwk}$ & SB1 & 14.29 & 0.28 & 123 & 4.95 & 20000 & 2.65 & 10 & 25 & 6.31 & 053855.83 & -690822.38 \\
\hline 697 & B1-2 & $\mathrm{Ve}$ & $\mathrm{SB} 1^{*}$ & 16.55 & 0.18 & 121 & - & - & - & - & - & - & 053857.28 & -690341.68 \\
\hline 705 & В 0.7 & V & SB1 & 16.43 & 0.07 & 87 & - & - & - & - & - & - & 053858.71 & -690647.10 \\
\hline 715 & B1 & $\mathrm{V}$ & SB1 & 16.64 & -0.10 & 116 & - & - & - & - & - & - & 053900.01 & -690139.76 \\
\hline 723 & B 0.5 & $\mathrm{~V}$ & SB1 & 16.19 & 0.16 & 63 & 4.50 & 27500 & 3.90 & - & - & - & 053902.85 & -690526.35 \\
\hline 730 & $\mathrm{~B} 1$ & $\operatorname{IV}(\mathrm{n})$ & $\mathrm{SB} 1^{*}$ & 15.41 & -0.10 & 248 & - & - & - & - & - & - & 053903.61 & -690018.98 \\
\hline 742 & B2 & V & SB1 & 16.93 & -0.02 & 60 & 3.73 & - & - & - & - & - & 053906.41 & -685658.70 \\
\hline 752 & B2 & V & SB2 & 16.48 & -0.12 & 194 & - & - & - & - & - & - & 053909.12 & -685747.26 \\
\hline 779 & B1 & II-Ib & SB1 & 15.46 & 0.19 & 47 & 4.73 & 23500 & 3.20 & 11 & 17 & 10.00 & 053921.53 & -690318.36 \\
\hline 784 & B1: & V & $\mathrm{SB} 1^{*}$ & 16.83 & 0.19 & 180 & - & - & - & - & - & - & 053924.22 & -690611.72 \\
\hline 827 & B 1.5 & $\mathrm{Ib}$ & SB1 & 15.34 & 0.31 & 52 & 5.03 & 21000 & 3.10 & 28 & 15 & 12.02 & 053939.27 & -691144.20 \\
\hline 837 & B1 & V & SB1 & 16.07 & -0.09 & 129 & - & - & - & - & - & - & 053941.25 & -685937.94 \\
\hline 847 & B $0.7-1$ & IIIne & $\mathrm{SB} 1^{*}$ & 15.48 & -0.06 & 258 & - & - & - & - & - & - & 053945.58 & -690426.23 \\
\hline 850 & $\mathrm{~B} 1$ & III & $\mathrm{SB} 1^{*}$ & 16.15 & 0.18 & $\leq 40$ & 4.34 & 24000 & 3.75 & - & - & - & 053951.16 & -691153.59 \\
\hline 874 & B1.5 & $\mathrm{IIIe}+$ & SB1 & 15.37 & 0.02 & 62 & 4.37 & - & - & - & - & - & 054010.33 & -690304.95 \\
\hline 877 & B1-3 & V-IIIe+ & $\mathrm{SB} 1^{*}$ & 16.36 & 0.18 & 267 & - & - & - & - & - & - & 054012.81 & -690910.30 \\
\hline 883 & B 0.5 & V & SB2 & 16.49 & 0.13 & 300 & - & - & - & - & - & - & 054018.03 & -690836.06 \\
\hline 888 & B 0.5 & V & SB1 & 16.18 & -0.07 & 76 & 4.18 & 27000 & 4.15 & - & - & - & 054022.62 & -690406.07 \\
\hline 890 & B2 & V & RV var & 16.13 & 0.02 & 160 & - & - & - & - & - & - & 054024.86 & -690944.14 \\
\hline 891 & B2 & V & SB1 & 16.48 & 0.07 & 55 & 4.04 & - & - & - & - & - & 054025.73 & -690630.78 \\
\hline
\end{tabular}

Notes. Definition of column headers: (1) VFTS identifier; (2) spectral types; (3) luminosity classes; (4) spectroscopic binary classification; (5) $V$-band magnitude; (6) $B$ - $V$ colour; (7) projected rotational velocity; (8) luminosity; (9) effective temperature; (10) logarithmic surface gravity; (11) spectroscopic mass; (12) evolutionary mass; (13) age; (14) right ascension; (15) declination. Evans et al. (2015) identified two SB2 systems, VFTS 112 and 199, that were classified as early B+early B (noted as 'EarlyBs' above); Evans et al. also identified VFTS 637 as having an early B-type companion. Projected rotational velocities, in the case of SB2 systems, should only be considered as upper limits since the majority of the systems were not classified as SB2 at the time of analysis from Dufton et al. (2013) and Garland et al. (2017). 
Table B2. Spectral lines used for RV measurements of each target. The systems where fewer epochs were used are generally the SB2 systems (see discussion in Section 3.1.1). He I $\lambda 4009$ and Mg II $\lambda 4481$ were used only in systems where we could not find a convincing period with our main set of lines and if we were able to perform an appropriate fit to them given the S/N of the spectra.

\begin{tabular}{|c|c|c|c|c|c|c|c|c|c|c|c|}
\hline VFTS & $\begin{array}{c}\mathrm{He} \mathrm{I} \\
\lambda 4009\end{array}$ & $\begin{array}{c}\mathrm{He} \text { I+ II } \\
\lambda 4026\end{array}$ & $\begin{array}{c}\mathrm{H} \delta \\
\lambda 4102\end{array}$ & $\begin{array}{c}\mathrm{He} \mathrm{I} \\
\lambda 4144\end{array}$ & $\begin{array}{c}\mathrm{H} \gamma \\
\lambda 4340\end{array}$ & $\begin{array}{c}\mathrm{He} \mathrm{I} \\
\lambda 4388\end{array}$ & $\begin{array}{c}\mathrm{He} \mathrm{I} \\
\lambda 4471\end{array}$ & $\begin{array}{c}\mathrm{Mg} \text { II } \\
\lambda 4481\end{array}$ & $\begin{array}{c}\text { Si III } \\
\lambda 4553\end{array}$ & $n$ lines & $n$ epochs \\
\hline 9 & - & $\mathrm{x}$ & - & $\mathrm{x}$ & $\mathrm{x}$ & $\mathrm{x}$ & $\mathrm{x}$ & - & - & 5 & 25 \\
\hline 15 & - & $\mathrm{x}$ & $\mathrm{x}$ & - & $\mathrm{x}$ & $\mathrm{x}$ & $\mathrm{x}$ & - & - & 5 & 25 \\
\hline 18 & - & $\mathrm{x}$ & $\mathrm{x}$ & $\mathrm{x}$ & - & $\mathrm{x}$ & - & - & - & 4 & 24 \\
\hline 27 & - & $\mathrm{x}$ & $\mathrm{x}$ & $\mathrm{x}$ & - & $\mathrm{x}$ & $\mathrm{x}$ & - & $\mathrm{x}$ & 6 & 25 \\
\hline 33 & - & $\mathrm{x}$ & - & $\mathrm{x}$ & - & $\mathrm{x}$ & $\mathrm{x}$ & - & - & 4 & 27 \\
\hline 37 & - & - & $\mathrm{x}$ & - & $\mathrm{x}$ & $\mathrm{x}$ & $\mathrm{x}$ & - & - & 4 & 20 \\
\hline 41 & - & $\mathrm{x}$ & $\mathrm{x}$ & $\mathrm{x}$ & - & $\mathrm{x}$ & $\mathrm{x}$ & - & - & 5 & 25 \\
\hline 97 & - & - & - & $\mathrm{x}$ & $\mathrm{x}$ & $\mathrm{x}$ & $\mathrm{x}$ & - & - & 4 & 24 \\
\hline 106 & - & $\mathrm{x}$ & - & - & $\mathrm{x}$ & - & $\mathrm{x}$ & - & - & 3 & 25 \\
\hline 112 & - & $\mathrm{x}$ & - & $\mathrm{x}$ & - & $\mathrm{x}$ & $\mathrm{x}$ & - & - & 4 & 15 \\
\hline 144 & - & $\mathrm{x}$ & $\mathrm{x}$ & $\mathrm{x}$ & - & - & $\mathrm{x}$ & - & - & 4 & 25 \\
\hline 146 & - & $\mathrm{x}$ & $\mathrm{x}$ & $\mathrm{x}$ & $\mathrm{x}$ & $\mathrm{x}$ & $\mathrm{x}$ & - & - & 6 & 20 \\
\hline 155 & - & $\mathrm{x}$ & - & $\mathrm{x}$ & $\mathrm{x}$ & $\mathrm{x}$ & $\mathrm{x}$ & - & - & 5 & 23 \\
\hline 157 & - & $\mathrm{x}$ & - & $\mathrm{x}$ & - & $\mathrm{x}$ & $\mathrm{x}$ & - & - & 4 & 24 \\
\hline 162 & - & $\mathrm{x}$ & $x$ & - & $x$ & $\mathrm{x}$ & - & - & - & 4 & 22 \\
\hline 179 & - & $\mathrm{x}$ & $\mathrm{x}$ & - & - & $x$ & $\mathrm{x}$ & - & - & 4 & 22 \\
\hline 189 & - & $\mathrm{x}$ & $\mathrm{x}$ & $x$ & $x$ & $\mathrm{x}$ & $\mathrm{x}$ & - & - & 6 & 27 \\
\hline 195 & - & $\mathrm{x}$ & $\mathrm{x}$ & $\mathrm{x}$ & - & $\mathrm{x}$ & $\mathrm{x}$ & - & $x$ & 6 & 23 \\
\hline 199 & - & $\mathrm{x}$ & - & $\mathrm{x}$ & - & $\mathrm{x}$ & $\mathrm{x}$ & - & - & 4 & 13 \\
\hline 204 & - & $\mathrm{x}$ & - & $\mathrm{x}$ & - & $\mathrm{x}$ & - & - & $x$ & 4 & 21 \\
\hline 206 & - & $\mathrm{x}$ & - & $\mathrm{x}$ & - & - & $\mathrm{x}$ & - & - & 3 & 14 \\
\hline 211 & - & $\mathrm{x}$ & - & $\mathrm{x}$ & - & $\mathrm{x}$ & $\mathrm{x}$ & - & - & 4 & 26 \\
\hline 213 & - & - & $x$ & - & - & $x$ & $x$ & - & - & 3 & 25 \\
\hline 215 & - & - & $\mathrm{x}$ & $x$ & $x$ & $x$ & $\mathrm{x}$ & - & - & 5 & 26 \\
\hline 218 & - & $x$ & - & - & - & $\mathrm{x}$ & $\mathrm{x}$ & - & - & 3 & 25 \\
\hline 225 & - & $\mathrm{x}$ & $x$ & $\mathrm{x}$ & $x$ & $\mathrm{x}$ & $\mathrm{x}$ & - & $\mathrm{x}$ & 7 & 25 \\
\hline 227 & - & $\mathrm{x}$ & $\mathrm{x}$ & $\mathrm{x}$ & $\mathrm{x}$ & $\mathrm{x}$ & $\mathrm{x}$ & - & - & 6 & 20 \\
\hline 240 & - & $\mathrm{x}$ & - & $\mathrm{x}$ & - & $\mathrm{x}$ & $x$ & - & - & 4 & 10 \\
\hline 246 & - & - & $x$ & $\mathrm{x}$ & $x$ & $\mathrm{x}$ & $\mathrm{x}$ & - & - & 5 & 23 \\
\hline 248 & - & $x$ & - & $\mathrm{x}$ & - & $x$ & $x$ & - & - & 4 & 17 \\
\hline 255 & - & $\mathrm{x}$ & - & $\mathrm{x}$ & - & $\mathrm{x}$ & - & - & - & 3 & 16 \\
\hline 257 & - & $\mathrm{x}$ & $x$ & $\mathrm{x}$ & - & - & $x$ & - & - & 4 & 22 \\
\hline 278 & - & $\hat{x}$ & - & $\mathrm{x}$ & $x$ & $x$ & $\mathrm{x}$ & - & - & 5 & 25 \\
\hline 291 & - & - & $\mathrm{x}$ & $\mathrm{x}$ & $\mathrm{x}$ & $\mathrm{x}$ & $\mathrm{x}$ & - & - & 5 & 26 \\
\hline 299 & - & $\mathrm{x}$ & $x$ & - & $x$ & - & - & - & - & 3 & 25 \\
\hline 305 & - & $\mathrm{x}$ & $\mathrm{x}$ & $\mathrm{x}$ & - & $x$ & $\mathrm{x}$ & - & - & 5 & 26 \\
\hline 324 & - & $\mathrm{x}$ & $\mathrm{x}$ & $\mathrm{x}$ & - & $\mathrm{x}$ & $\mathrm{x}$ & - & $\mathrm{x}$ & 6 & 28 \\
\hline 334 & - & $\mathrm{x}$ & $\mathrm{x}$ & - & $x$ & $\mathrm{x}$ & $\mathrm{x}$ & - & - & 5 & 22 \\
\hline 337 & - & - & $\mathrm{x}$ & $\mathrm{x}$ & $\mathrm{x}$ & $x$ & $\mathrm{x}$ & - & - & 5 & 19 \\
\hline 342 & - & $\mathrm{x}$ & $\mathrm{x}$ & $\mathrm{x}$ & $\mathrm{x}$ & $\mathrm{x}$ & $\mathrm{x}$ & - & - & 6 & 24 \\
\hline 351 & - & $x$ & $\mathrm{x}$ & $x$ & $x$ & $\mathrm{x}$ & $x$ & - & - & 6 & 25 \\
\hline 359 & - & $\mathrm{x}$ & $\mathrm{x}$ & - & $\mathrm{x}$ & $\mathrm{x}$ & - & - & - & 4 & 23 \\
\hline 364 & - & $\mathrm{x}$ & - & $x$ & - & $\mathrm{x}$ & $x$ & - & - & 4 & 18 \\
\hline 383 & - & - & - & $\mathrm{x}$ & - & $\mathrm{x}$ & $\mathrm{x}$ & - & - & 3 & 15 \\
\hline 388 & - & - & $x$ & $\mathrm{x}$ & - & - & $\mathrm{x}$ & - & - & 3 & 23 \\
\hline 391 & - & - & - & $\mathrm{x}$ & $\mathrm{x}$ & $x$ & $\mathrm{x}$ & - & - & 4 & 22 \\
\hline 396 & - & - & $x$ & $\mathrm{x}$ & - & $\mathrm{x}$ & $\mathrm{x}$ & - & - & 4 & 22 \\
\hline 430 & - & $\mathrm{x}$ & - & $\mathrm{x}$ & - & $x$ & $x$ & - & $\mathrm{x}$ & 5 & 27 \\
\hline 434 & - & $\mathrm{x}$ & - & $\mathrm{x}$ & - & $\mathrm{x}$ & $\mathrm{x}$ & - & - & 4 & 24 \\
\hline 442 & - & $\mathrm{x}$ & $x$ & $\mathrm{x}$ & - & $\mathrm{x}$ & - & - & - & 4 & 24 \\
\hline 501 & - & $\mathrm{x}$ & - & $\mathrm{x}$ & $\mathrm{x}$ & - & - & - & $\mathrm{x}$ & 4 & 25 \\
\hline 520 & - & $\mathrm{x}$ & - & $\mathrm{x}$ & - & $\mathrm{x}$ & - & - & - & 3 & 17 \\
\hline 534 & - & $\mathrm{x}$ & $\mathrm{x}$ & $\mathrm{x}$ & - & - & $\mathrm{x}$ & - & $\mathrm{x}$ & 5 & 26 \\
\hline 575 & - & $\mathrm{x}$ & - & $\mathrm{x}$ & - & $x$ & $x$ & - & $\mathrm{x}$ & 5 & 28 \\
\hline 576 & - & $\mathrm{x}$ & - & $\mathrm{x}$ & - & $\mathrm{x}$ & $\mathrm{x}$ & - & $\mathrm{x}$ & 5 & 23 \\
\hline 589 & - & - & - & $\mathrm{x}$ & - & $\mathrm{x}$ & - & - & $\mathrm{x}$ & 3 & 18 \\
\hline 591 & $x$ & - & - & $\mathrm{x}$ & - & $\mathrm{x}$ & - & - & $\mathrm{x}$ & 4 & 29 \\
\hline 606 & - & $\mathrm{x}$ & $\mathrm{x}$ & $\mathrm{x}$ & - & - & $\mathrm{x}$ & - & - & 4 & 24 \\
\hline 637 & - & $\mathrm{x}$ & - & $\mathrm{x}$ & - & $\mathrm{x}$ & $\mathrm{x}$ & - & - & 4 & 15 \\
\hline 662 & - & - & $x$ & $\mathrm{x}$ & - & - & - & $\mathrm{x}$ & - & 3 & 23 \\
\hline
\end{tabular}


J. I. Villaseñor et al.

Table B2 - continued

\begin{tabular}{|c|c|c|c|c|c|c|c|c|c|c|c|}
\hline VFTS & $\begin{array}{c}\mathrm{He} \mathrm{I} \\
\lambda 4009\end{array}$ & $\begin{array}{c}\mathrm{He} \text { I+ II } \\
\lambda 4026\end{array}$ & $\begin{array}{c}\mathrm{H} \delta \\
\lambda 4102\end{array}$ & $\begin{array}{c}\mathrm{He} \mathrm{I} \\
\lambda 4144\end{array}$ & $\begin{array}{c}\mathrm{H} \gamma \\
\lambda 4340\end{array}$ & $\begin{array}{c}\mathrm{He} \mathrm{I} \\
\lambda 4388\end{array}$ & $\begin{array}{c}\mathrm{He} \mathrm{I} \\
\lambda 4471\end{array}$ & $\begin{array}{c}\mathrm{Mg} \text { II } \\
\lambda 4481\end{array}$ & $\begin{array}{c}\text { Si III } \\
\lambda 4553\end{array}$ & $n$ lines & $n$ epochs \\
\hline 665 & - & - & $\mathrm{x}$ & $\mathrm{x}$ & $\mathrm{x}$ & - & $\mathrm{x}$ & - & - & 4 & 21 \\
\hline 686 & $\mathrm{x}$ & - & - & $\mathrm{x}$ & - & $\mathrm{x}$ & - & - & - & 3 & 19 \\
\hline 687 & - & $\mathrm{x}$ & - & $\mathrm{x}$ & $\mathrm{x}$ & $\mathrm{x}$ & $\mathrm{x}$ & - & $\mathrm{x}$ & 6 & 27 \\
\hline 697 & - & $\mathrm{x}$ & - & $\mathrm{x}$ & - & $\mathrm{x}$ & - & - & - & 3 & 25 \\
\hline 705 & - & $\mathrm{x}$ & $\mathrm{x}$ & - & $\mathrm{x}$ & $\mathrm{x}$ & $\mathrm{x}$ & - & - & 5 & 25 \\
\hline 715 & - & $\mathrm{x}$ & $\mathrm{x}$ & $\mathrm{x}$ & - & $\mathrm{x}$ & $\mathrm{x}$ & - & - & 5 & 23 \\
\hline 718 & - & $\mathrm{x}$ & $\mathrm{x}$ & $\mathrm{x}$ & - & $\mathrm{x}$ & $\mathrm{x}$ & - & - & 5 & 27 \\
\hline 719 & - & $\mathrm{x}$ & $\mathrm{x}$ & $\mathrm{x}$ & $\mathrm{x}$ & $\mathrm{x}$ & $\mathrm{x}$ & - & - & 6 & 25 \\
\hline 723 & - & $\mathrm{x}$ & $\mathrm{x}$ & $\mathrm{x}$ & - & $\mathrm{x}$ & $\mathrm{x}$ & - & $\mathrm{x}$ & 6 & 21 \\
\hline 730 & $\mathrm{x}$ & $\mathrm{x}$ & - & $\mathrm{x}$ & - & - & - & - & - & 3 & 18 \\
\hline 742 & - & $\mathrm{x}$ & - & - & $\mathrm{x}$ & $\mathrm{x}$ & $\mathrm{x}$ & - & - & 4 & 23 \\
\hline 752 & - & $\mathrm{x}$ & - & $\mathrm{x}$ & - & $\mathrm{x}$ & $\mathrm{x}$ & - & - & 4 & 16 \\
\hline 779 & - & $\mathrm{x}$ & - & $\mathrm{x}$ & $\mathrm{x}$ & $\mathrm{x}$ & $\mathrm{x}$ & - & $\mathrm{x}$ & 6 & 25 \\
\hline 784 & - & - & $\mathrm{x}$ & - & - & $\mathrm{x}$ & - & - & - & 2 & 21 \\
\hline 788 & - & $\mathrm{x}$ & $\mathrm{x}$ & $\mathrm{x}$ & $\mathrm{x}$ & $\mathrm{x}$ & $x$ & - & - & 6 & 24 \\
\hline 792 & $x$ & $\mathrm{x}$ & - & $x$ & - & $\mathrm{x}$ & - & $\mathrm{x}$ & - & 5 & 16 \\
\hline 799 & - & $x$ & $x$ & - & $x$ & - & - & $x$ & $\mathrm{x}$ & 5 & 22 \\
\hline 821 & - & $\mathrm{x}$ & - & $x$ & - & $x$ & $\mathrm{x}$ & - & - & 4 & 24 \\
\hline 827 & - & $\mathrm{x}$ & - & $x$ & $\mathrm{x}$ & $\mathrm{x}$ & $\mathrm{x}$ & - & $\mathrm{x}$ & 6 & 26 \\
\hline 837 & - & $\mathrm{x}$ & - & $\mathrm{x}$ & $\mathrm{x}$ & $\mathrm{x}$ & $\mathrm{x}$ & - & - & 5 & 24 \\
\hline 847 & $x$ & - & - & $\mathrm{x}$ & - & - & - & - & - & 2 & 26 \\
\hline 850 & - & - & - & $x$ & $\mathrm{x}$ & $\mathrm{x}$ & $\mathrm{x}$ & - & - & 4 & 21 \\
\hline 874 & - & $x$ & $\mathrm{x}$ & - & $\mathrm{x}$ & $\mathrm{x}$ & - & - & $\mathrm{x}$ & 5 & 25 \\
\hline 877 & - & $\mathrm{x}$ & - & $x$ & $\mathrm{x}$ & - & $x$ & - & - & 4 & 19 \\
\hline 883 & - & $\mathrm{x}$ & - & $x$ & - & $x$ & $\mathrm{x}$ & - & - & 4 & 10 \\
\hline 888 & - & $\mathrm{x}$ & $\mathrm{x}$ & $\mathrm{x}$ & $\mathrm{x}$ & $\mathrm{x}$ & $\mathrm{x}$ & - & - & 6 & 22 \\
\hline 890 & $\mathrm{x}$ & $x$ & $\mathrm{x}$ & - & $\mathrm{x}$ & $\mathrm{x}$ & $\mathrm{x}$ & - & - & 6 & 21 \\
\hline 891 & - & $\mathrm{x}$ & - & $x$ & - & $\mathrm{x}$ & $\mathrm{x}$ & - & - & 4 & 25 \\
\hline
\end{tabular}









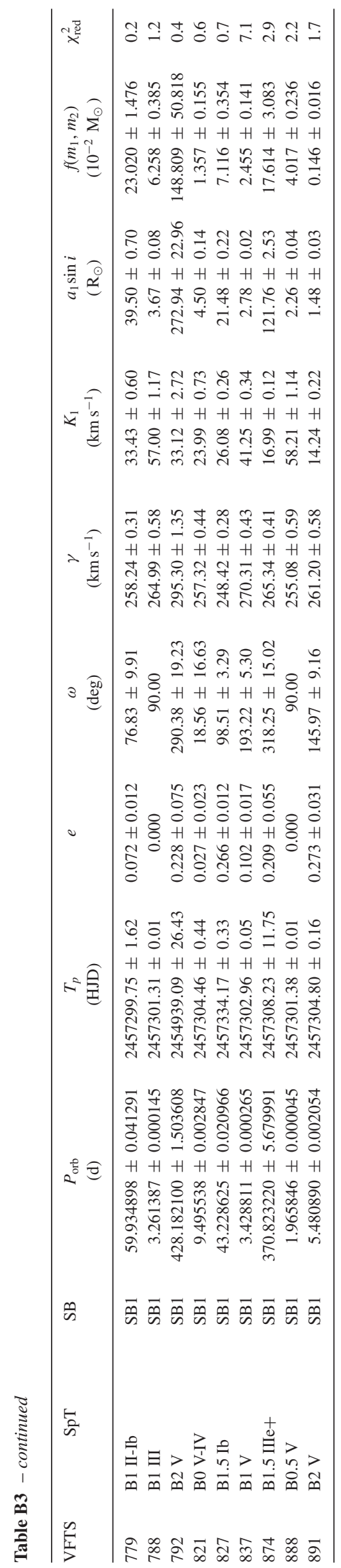




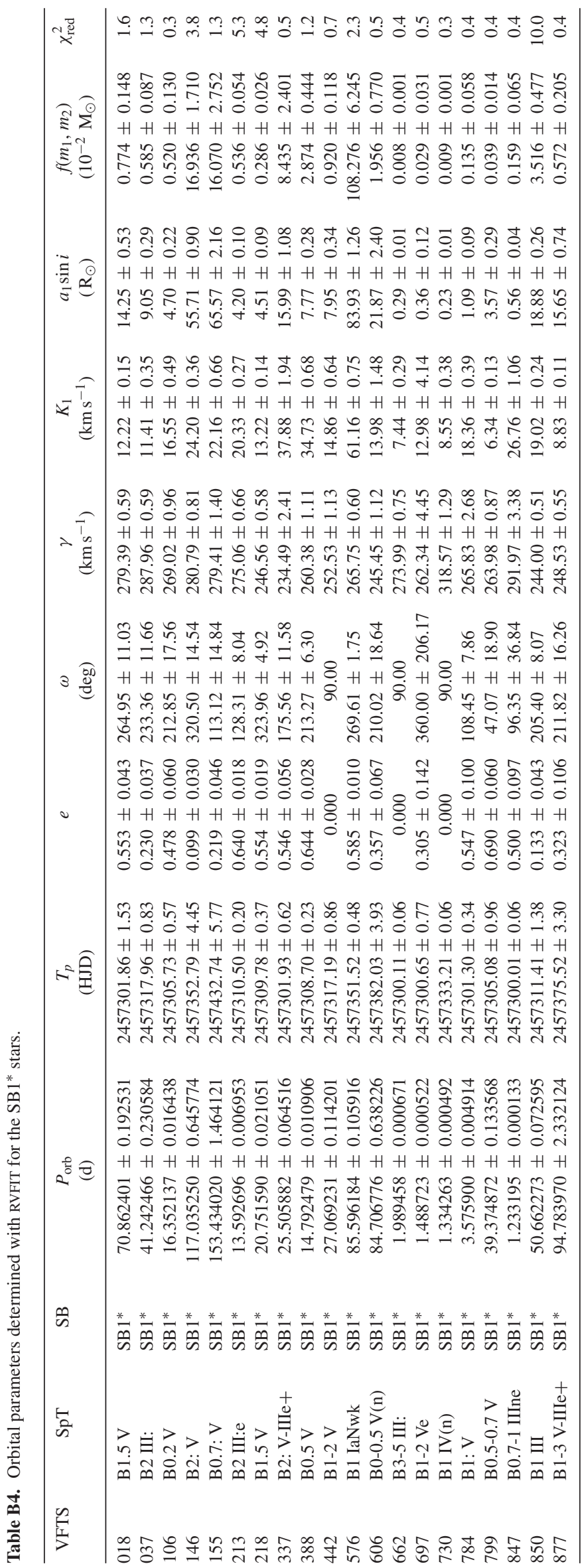


Table B5. Orbital parameters determined with RVFIT for the SB2 sample.

\begin{tabular}{|c|c|c|c|c|c|c|c|c|c|}
\hline VFTS & $\mathrm{SB}$ & $\begin{array}{l}P_{\text {orb }} \\
\text { (d) }\end{array}$ & $\begin{array}{c}T_{p} \\
\text { (HJD) }\end{array}$ & $e$ & $\begin{array}{c}\omega \\
(\mathrm{deg})\end{array}$ & $\begin{array}{c}\gamma \\
\left(\mathrm{km} \mathrm{s}^{-1}\right)\end{array}$ & $\begin{array}{c}K_{1} \\
\left(\mathrm{~km} \mathrm{~s}^{-1}\right)\end{array}$ & $\begin{array}{c}K_{2} \\
\left(\mathrm{~km} \mathrm{~s}^{-1}\right)\end{array}$ & $\chi_{\text {red }}^{2}$ \\
\hline 112 & SB2 & $674083 \pm 0.000030$ & 2457300.4 & 0.000 & 0 & $54.02 \pm 1.24$ & $222.35 \pm 1.66$ & $266.80 \pm 2.58$ & 4.8 \\
\hline 206 & SB2 & $7.556457 \pm 0.000906$ & $2457305.59 \pm 0.27$ & $029 \pm 0.012$ & $315.73 \pm 13.02$ & $262.54 \pm 0.91$ & $97.10 \pm 1.94$ & $108.62 \pm 1.16$ & 5.4 \\
\hline 240 & SB2 & $1.377795 \pm 0.000060$ & $2457300.43 \pm 0.04$ & $.096 \pm 0.014$ & $0.00 \pm 8.59$ & $255.51 \pm 1.27$ & $142.38 \pm 3.07$ & $147.09 \pm 1.76$ & 7.5 \\
\hline 248 & SB2 & $2.494240 \pm 0.000058$ & $2457299.86 \pm 0.02$ & $0.166 \pm 0.007$ & $183.46 \pm 2.40$ & $278.48 \pm 0.90$ & 181.55 & $238.41 \pm 1.89$ & 11.8 \\
\hline 255 & SB2 & $2.844891 \pm 0.000100$ & $2457335.35 \pm 0.06$ & $0.024 \pm 0.009$ & $111.52 \pm 7.67$ & $251.27 \pm 1.26$ & $193.92 \pm 2.16$ & $200.14 \pm 2.28$ & 8.2 \\
\hline 520 & SB2 & $2.947548 \pm 0.000123$ & $2457300.83 \pm 0.05$ & $0.142 \pm 0.013$ & $309.17 \pm 6.25$ & $256.74 \pm 1.12$ & $114.60 \pm 1.31$ & $176.82 \pm 3.09$ & 8.0 \\
\hline 589 & SB2 & $7.629761 \pm 0.000505$ & $2457332.83 \pm 0.03$ & $0.267 \pm 0.006$ & $65.56 \pm 1.61$ & $276.33 \pm 0.50$ & $97.11 \pm 0.62$ & $121.52 \pm 1.15$ & 7.0 \\
\hline 637 & SB2 & $1.628692 \pm 0.000033$ & $2457300.91 \pm 0.03$ & $0.026 \pm 0.008$ & $97.80 \pm 7.68$ & $240.20 \pm 1.12$ & $214.02 \pm 1.69$ & $241.65 \pm 2.65$ & 6.1 \\
\hline 686 & SB2 & $16.869613 \pm 0.002899$ & $2457304.83 \pm 0.13$ & $0.311 \pm 0.007$ & $117.54 \pm 2.18$ & $253.69 \pm$ & $71.56 \pm 0.68$ & $101.93 \pm 1.89$ & 14.9 \\
\hline 752 & SB2 & $07603 \pm 0.000028$ & $2457300.07 \pm 0.00$ & 0.000 & 90.00 & $248.93 \pm 0.70$ & $127.63 \pm 1.03$ & $140.60 \pm 1.31$ & 2.4 \\
\hline 883 & SB2 & $3.495192 \pm 0.000330$ & $2457302.86 \pm 0.02$ & $0.359 \pm 0.013$ & $161.48 \pm 3.66$ & $248.28 \pm 1.80$ & $170.74 \pm 3.21$ & $226.81 \pm 3.87$ & 7.1 \\
\hline
\end{tabular}

Table B6. Derived orbital parameters for the SB2 sample.

\begin{tabular}{|c|c|c|c|c|c|c|c|}
\hline VFTS & SpT & $\begin{array}{c}M_{1} \sin ^{3} i \\
\quad\left(\mathrm{M}_{\odot}\right)\end{array}$ & $\begin{array}{c}M_{2} \sin ^{3} i \\
\quad\left(\mathrm{M}_{\odot}\right)\end{array}$ & $\begin{array}{c}q \\
\left(M_{2} / M_{1}\right)\end{array}$ & $\begin{array}{c}a_{1} \sin i \\
\left(\mathrm{R}_{\odot}\right)\end{array}$ & $\begin{array}{c}a_{2} \sin i \\
\left(\mathrm{R}_{\odot}\right)\end{array}$ & $\begin{array}{l}a \sin i \\
\left(\mathrm{R}_{\odot}\right)\end{array}$ \\
\hline 112 & EarlyBs & $11.07 \pm 0.24$ & $9.23 \pm 0.16$ & $0.83 \pm 0.01$ & $7.36 \pm 0.05$ & $8.83 \pm 0.09$ & $16.19 \pm 0.10$ \\
\hline 206 & B3 III: & $3.59 \pm 0.10$ & $3.21 \pm 0.13$ & $0.89 \pm 0.02$ & $14.50 \pm 0.29$ & $16.22 \pm 0.17$ & $30.71 \pm 0.34$ \\
\hline 240 & B1-2 V & $1.74 \pm 0.06$ & $1.68 \pm 0.07$ & $0.97 \pm 0.02$ & $3.86 \pm 0.08$ & $3.99 \pm 0.05$ & $7.85 \pm 0.10$ \\
\hline 248 & B2: V & $10.42 \pm 0.19$ & $7.93 \pm 0.13$ & $0.76 \pm 0.01$ & $8.83 \pm 0.06$ & $11.59 \pm 0.09$ & $20.42 \pm 0.11$ \\
\hline 383 & B0.5: V & $1.25 \pm 0.03$ & $0.88 \pm 0.03$ & $0.70 \pm 0.02$ & $4.21 \pm 0.09$ & $6.01 \pm 0.04$ & $10.23 \pm 0.10$ \\
\hline 520 & B1: V & $4.45 \pm 0.18$ & $2.88 \pm 0.09$ & $0.65 \pm 0.01$ & $6.61 \pm 0.08$ & $10.20 \pm 0.18$ & $16.81 \pm 0.19$ \\
\hline 589 & B $0.5 \mathrm{~V}$ & $4.11 \pm 0.09$ & $3.28 \pm 0.06$ & $0.80 \pm 0.01$ & $14.11 \pm 0.09$ & $17.66 \pm 0.17$ & $31.78 \pm 0.19$ \\
\hline 637 & B1-2 V+EarlyB & $8.46 \pm 0.20$ & $7.49 \pm 0.14$ & $0.89 \pm 0.01$ & $6.89 \pm 0.05$ & $7.78 \pm 0.09$ & $14.66 \pm 0.10$ \\
\hline 686 & B0.7 III & $4.61 \pm 0.19$ & $3.23 \pm 0.09$ & $0.70 \pm 0.01$ & $22.68 \pm 0.22$ & $32.31 \pm 0.60$ & $54.99 \pm 0.64$ \\
\hline 752 & B2 V & $1.48 \pm 0.03$ & $1.34 \pm 0.02$ & $0.91 \pm 0.01$ & $3.55 \pm 0.03$ & $3.91 \pm 0.04$ & $7.46 \pm 0.05$ \\
\hline
\end{tabular}


Table B7. Additional stars observed as part of the BBC programme.

\begin{tabular}{|c|c|c|c|c|}
\hline VFTS & Classification & $V$ & $B-V$ & Comments \\
\hline \multicolumn{5}{|c|}{ Candidate O-type runaways: } \\
\hline 072 & O2 V-III(n)((f*)) & 13.70 & -0.14 & \\
\hline 138 & O9 Vn & 15.63 & -0.09 & \\
\hline 249 & $\mathrm{O} 8 \mathrm{Vn}$ & 15.52 & -0.03 & \\
\hline 285 & O7.5 Vnnn & 15.63 & -0.06 & \\
\hline 356 & O6: V(n)z & 15.87 & 0.16 & \\
\hline 660 & O9.5 Vnn & 15.92 & 0.03 & \\
\hline 706 & O6-7 Vnnz & 15.77 & 0.14 & \\
\hline 722 & O7 Vnnz & 15.04 & -0.13 & \\
\hline 746 & O6 Vnn & 15.38 & 0.12 & \\
\hline 751 & O7-8 Vnnz & 16.32 & 0.15 & \\
\hline 755 & $\mathrm{O} 3 \mathrm{Vn}((\mathrm{f} *))$ & 15.04 & 0.14 & \\
\hline 768 & O8 Vn & 16.10 & 0.20 & \\
\hline 770 & O7 Vnn & 15.79 & 0.08 & \\
\hline \multicolumn{5}{|c|}{ Other VFTS targets: } \\
\hline 091 & O9.5 IIIn & 15.98 & 0.20 & N-enriched (Grin et al. 2017) \\
\hline 102 & O9: Vnnne+ & 15.70 & 0.35 & Extreme rotator (Dufton et al. 2011) \\
\hline 267 & O3 III-I(n)f* & 13.49 & -0.05 & N-enriched (Grin et al. 2017) \\
\hline 298 & B1-2 V-IIIe+ & 16.68 & 0.24 & Candidate runaway (Evans et al. 2015) \\
\hline 328 & O9.5 III(n) & 15.90 & -0.11 & N-enriched (Grin et al. 2017) \\
\hline 358 & B0.5: V & 16.87 & 0.00 & Candidate runaway (Evans et al. 2015) \\
\hline 368 & B1-3 V & 16.68 & 0.01 & Candidate runaway (Evans et al. 2015) \\
\hline 399 & O9 IIIn & 15.83 & 0.08 & X-ray bright (Clark et al. 2015) \\
\hline 456 & Onn & 15.46 & 0.13 & Rapid rotator (Ramírez-Agudelo et al. 2013) \\
\hline 467 & $\mathrm{~B} 1-2 \mathrm{Ve}+$ & 16.91 & 0.38 & Candidate runaway (Evans et al. 2015) \\
\hline 574 & O9.5 IIIn & 15.89 & -0.12 & N-enriched (Grin et al. 2017) \\
\hline 698 & mid-B + early-B & 13.68 & 0.44 & Peculiar B[e]-like system (Dunstall et al. 2012) \\
\hline 703 & O7: V: + O8: V: & 16.91 & 0.30 & O-type SB2 (not observed by Almeida et al. 2017) \\
\hline 704 & $\mathrm{O} 9.2 \mathrm{~V}(\mathrm{n})$ & 16.76 & -0.07 & Moderately rapid rotator $\left(240-300 \mathrm{~km} \mathrm{~s}^{-1}\right)$ \\
\hline
\end{tabular}

Note. Classifications are from Walborn et al. (2014) and Evans et al. (2015), for O- and B-type spectra, respectively. Photometry is from Evans et al. (2011).

This paper has been typeset from a $\mathrm{T}_{\mathrm{E}} \mathrm{X} / \mathrm{LT} \mathrm{E} \mathrm{X}$ file prepared by the author. 